\title{
Exposures and behavioral responses to wildfire smoke
}

Marshall Burke ${ }^{1,2,3, \diamond}$, Sam Heft-Neal ${ }^{2}$, Jessica $\mathrm{Li}^{2}$, Anne Driscoll ${ }^{2}$, Patrick Baylis ${ }^{4}$, Matthieu Stigler $^{2}$, Joakim Weill ${ }^{5}$, Jennifer Burney ${ }^{6}$, Jeff Wen ${ }^{1}$, Marissa Childs ${ }^{7}$, Carlos F Gould ${ }^{1}$

${ }^{1}$ Dept. of Earth System Science, Stanford University

${ }^{2}$ Center on Food Security and the Environment, Stanford University

${ }^{3}$ National Bureau of Economic Research

${ }^{4}$ Dept. of Economics, University of British Columbia

${ }^{5}$ Dept. of Agricultural and Resource Economics, UC-Davis

${ }^{6}$ Global Policy School, UC-San Diego

${ }^{7}$ Emmett Interdisciplinary Program in Environment and Resources, Stanford University

October 11, 2021

The paper is a non-peer reviewed preprint submitted to EarthArXiv. It has also been submitted for publication in a peer reviewed journal, but has yet to be formally accepted for publication.

$\diamond$ Communicating author: mburke@stanford.edu

Twitter: MarshallBBurke

\begin{abstract}
:
The impacts of environmental change on human outcomes often depend on local exposures and behavioral responses that are challenging to observe with traditional administrative or sensor data. We show how data from private pollution sensors, cell phones, social media posts, and internet search activity yield new insights on exposures and behavioral responses during large wildfire smoke events across the US, a rapidly-growing environmental stressor. Health-protective behavior, mobility, and sentiment all respond to increasing ambient wildfire smoke concentrations, but responses differ by income. Indoor pollution monitors provide starkly different estimates of likely personal exposure during smoke events than would be inferred from traditional ambient outdoor sensors, with similar outdoor pollution levels generating $>\mathbf{2 0 x}$ differences in average indoor $\mathbf{P M}_{2.5}$ concentrations. Our results suggest that the current policy reliance on self protection to mitigate health risks in the face of rising smoke exposure will result in modest and unequal benefits.
\end{abstract}


A large body of scientific evidence documents how environmental exposures can substantially shape human outcomes. For instance, poor air quality is estimated to kill millions of people per year (1), warming temperatures lead to more crime and less economic output (2), and exposure to lead reduces educational achievement (3). Effects can vary substantially across subgroups: air pollution is more harmful to health in poorer US counties (4), warming temperatures have more negative effects on economic output in already hot locations (5), and lead exposures appear to have larger effects in already disadvantaged households (6). Standard models of behavior in both public health and economics suggest the magnitude and variation of these effects in part reflect choices that individuals make or are unable to make regarding their exposures, and that the choice sets available to individuals are in turn reflective of individuals' knowledge, circumstances, and preferences $(7,8)$. Unfortunately, these decision-making components, as well as their behavioral outcomes, are typically hard to observe at scale. This makes it difficult to understand why a given environmental exposure generates the effect it does, why this effect might differ across groups, and whether and how policy should respond.

Here we show how combining traditional sensor and survey data with information from nontraditional distributed sensors - including data from private outdoor and indoor pollution sensors, cell phones, social media posts, and internet search activity - can generate population-scale insights into people's knowledge, preferences, and choices regarding a changing environment, and into how economic circumstances shape their choice set. We focus on understanding responses to wildfire smoke, a rapidly growing environmental stressor throughout much of the US and internationally. Annual area burned by wildfires in the US has more than doubled in recent decades, a result of a century of fire suppression and a warming climate that has left the resulting abundant fuel much more flammable (9). This increase in fire activity has led to substantial increases in average smoke exposure across the continental US, potentially reversing decades of improvements in air quality (10). Absent substantial intervention, these trends are expected to continue and perhaps accelerate in a warming climate (11-13).

A growing literature has begun to document myriad health impacts of ambient wildfire smoke exposure (14-18). As with impacts of other environmental stressors, the magnitude of these health impacts likely depend on behaviors and individual-specific exposures that are often poorly measured and understood. In particular, recent wildfire case studies suggest that individuals vary in their knowledge and beliefs about their own exposures and about the risk that these exposures pose to their health $(19,20)$. Documented heterogeneity in the impacts of wildfire smoke exposure or exposure to other particulates $(4,18,21-23)$ suggest that both socioeconomic circumstances and previous exposures could also constrain behavior in important ways. Understanding which features matter most is consequential for policy design: impacts driven by a lack of aware- 
ness of one's exposure, for instance, call for different interventions than impacts driven by an inability to protect oneself from a known exposure. Such understanding is particularly important for wildfire, given that current policy approaches to risk mitigation focus on the private provision of protection - i.e., asking individuals to stay indoors, limit infiltration, and purchase protective technologies (24).

To better understand exposures, behavioral responses, and outcomes in the face of rapidly changing wildfire risk, we first develop measures of daily exposure to ambient $\mathrm{PM}_{2.5}$ from wildfire smoke, as this exposure itself is not directly measured by existing pollution sensors. To do so we combine long time series of regulatory ground monitor data on $\mathrm{PM}_{2.5}$ concentrations from US Environmental Protection Agency (EPA) pollution monitors with satellite-derived estimates of smoke exposure. To isolate wildfire-smoke-derived $\mathrm{PM}_{2.5}$ from other sources of $\mathrm{PM}_{2.5}$, we define smoke $\mathrm{PM}_{2.5}$ as location-, month-, and period-specific anomalous $\mathrm{PM}_{2.5}$ on days in which satellites indicated that smoke was overhead (Methods). Resulting ambient smoke $\mathrm{PM}_{2.5}$ exposure data cover 970 US counties that contain $\sim 80 \%$ of US population (Fig S2), and display wide spatial and temporal variability (Fig 1). Satellite-based counts of the annual number of dense smoke plumes overhead have trended upward in the last decade throughout most of the continental US, particularly in the West (Fig 1a), which we estimate have helped drive rapid increases in estimated annual smoke $\mathrm{PM}_{2.5}$ across the country and in days with extreme $\mathrm{PM}_{2.5}$ due to smoke (Fig 1b-c).

We merge these daily wildfire-smoke-derived data with multiple high-frequency datasets that are measured at population scale and shed light on individuals' knowledge, beliefs and behaviors during wildfires. To study individuals' awareness of their exposure, we first analyze locationspecific variation in search query behavior related to smoke exposure. We use public data on specific search queries (e.g. 'air quality') from Google Trends, which provides normalized data on search term popularity at the weekly level across "designated market areas" (roughly, groups of counties; see Methods). We interpret a purposeful search for information related to smoke exposure as evidence that an individual is aware she is being exposed and that her exposure level is worth learning about - what we call "salience".

Second, we study individuals' preferences and sentiments regarding wildfire smoke exposure. Such preferences underlie standard theoretical models of choice behavior but are hard to observe directly and at scale. Past work has shown that social media behavior can be a sensitive and accurate tool for understanding individuals' preferences and sentiments toward what is happening around them (25), including a changing environment $(26,27)$. Following this earlier work, we analyze $\sim 1.7$ billion georeferenced Twitter updates ("tweets") posted since 2016 using natural 
language processing algorithms that extract information on the sentiment revealed in each tweet (28) (Methods). This approach has been validated at population scale against self-reported measures of emotional state (25), and complements earlier work that used Twitter to directly measure wildfire activity (29) and infer smoke concentrations (30).

Third, we again use Google search queries to study whether individuals sought information regarding specific health-protective actions, analyzing item-specific search terms like "air filter", "air purifier", or "smoke mask". While we can only observe whether individuals eventually purchased these items in the case of PurpleAir monitors as described below, such search behavior can be interpreted, at a minimum, as evidence of an individual's belief that health-protective options exist. Evidence from other settings suggests that search activity is predictive of future behavior, including consumer purchases $(31,32)$.

Fourth, we use smartphone-derived location data to study whether individuals altered their physical movements during periods of smoke exposure. Short-term migration in response to other environmental stress (e.g. hurricanes) is common, and is a plausible avenue by which individuals or households could seek to limit exposure to wildfire smoke. We study both the share of people estimated to be completely at home, and the share estimated to be completely away from their home, on days or weeks of smoke exposure.

We combine each measure with our smoke $\mathrm{PM}_{2.5}$ data and analyze the effect of smoke on each outcome using panel fixed effects estimators that exploit local temporal variation in both exposures and outcomes. While long-term exposure to wildfire smoke shows clear spatial patterns and temporal trends (Fig 1), local-level variation in daily exposure is highly random, and panel estimators - which are commonly employed in related environmental settings (2) - plausibly isolate the impact of variation in smoke exposure from other time-invariant and time-varying factors that could be correlated with both smoke exposure and outcomes, including potential confounding from COVID-19 (Methods). To ensure that we are measuring the impact of wildfire smoke and not simply proximity to wildfire itself, we develop measures of distance to the nearest active wildfire and analyze whether responses differ by fire proximity.

Finally, we analyze how ambient outdoor smoke $\mathrm{PM}_{2.5}$ infiltrates into the indoor home environment, and whether behaviors and circumstances shape this infiltration. Understanding indoor concentrations is critical, as individuals in the US spend the vast majority of their time indoors. Using data from the American Time Use Survey, we calculate that Americans on average spend $>70 \%$ of their time indoors at home, with higher shares for lower-income and elderly individuals and overall shares trending up over time (Fig S1). Personal integrated exposure to variation in 
ambient exposure is then likely substantially mediated by characteristics of home and work environments that are hard to observe (33-35), and these differences could in turn affect outcomes (36-39). If socioeconomic or demographic variables shape indoor environments in ways that affect exposures, as has been hypothesized (37), then exposure levels or policy choices that appear equitable based on traditional outdoor measures could obscure large disparities in realized exposures.

We assemble and harmonize hourly data from 1,520 indoor PurpleAir air pollution monitors that individuals have put in their single-family homes across the US and use nearby outdoor PurpleAir monitors to construct outdoor $\mathrm{PM}_{2.5}$ concentrations at each home (Methods). To estimate infiltration, we use distributed lag or lagged-dependent variable panel regression to estimate the marginal increase in indoor $\mathrm{PM}_{2.5}$ when outdoor $\mathrm{PM}_{2.5}$ increases by one unit (i.e., OIndoor $P M_{2.5} / \partial$ Outdoor $P M_{2.5}$ ), controlling flexibly for time of day, day of week, and month of sample (Methods). We estimate models that pool all indoor monitors as well as monitor-specific models, and study how infiltration differs as a function of household and neighborhood characteristics. Our approach complements recent work using PurpleAir to study infiltration generally (40) and during wildfires specifically (33), though our estimation approach offers advantages relative to the latter such as robustness to indoor pollution sources and to diurnal patterns in infiltrationrelevant behaviors (Methods).

The timeliness and granularity of novel passive distributed sensor data needs to be weighed against its potential non-representativeness, as the latter can bias population-scale inferences. Our search data and mobility data are likely our most representative, as the vast majority of Americans use the internet regularly and most own and use smartphones. Twitter users are less representative on average, but Twitter-derived sentiment measures have been shown to validate well against population emotional state, and related work shows that the response of sentiment to environmental stress mirrors that measured in representative survey data. PurpleAir data are least representative of our datasets, with wealthier and more educated households more likely to own monitors; however, as discussed below, socioeconomic and demographic information does not appear strongly predictive of infiltration. See Methods for more detailed discussion of sample representativeness.

\section{Results}

Increases in wildfire-derived ambient $\mathrm{PM}_{2.5}$ exposure lead to an increase in popularity for air quality-related search terms (Fig 2a), with even small increases above zero exposure appearing 
salient (Fig 2a). Results are robust to alternate air-quality-related search terms and to using analogous search terms in Spanish (Table S3), are not driven by proximity to active wildfires, and are robust to inclusion of weather controls or alternate fixed effects (Table S4). Our results are consistent with interview-based evidence that found that individuals who perceived they were being exposed to smoke often used internet-based sources to confirm their perceptions (19). The salience of ambient exposure at low levels is also somewhat reassuring given recent evidence of health impacts for sensitive populations even at very low levels of ambient exposure $(18,41)$.

We find that exposure to ambient smoke $\mathrm{PM}_{2.5}$ makes people unhappier, as measured in an automated sentiment analysis of 5 years of tweets across the US. Expressed sentiment in tweets declines roughly linearly above smoke $\mathrm{PM}_{2.5}$ exposures of $\sim 20 \mu \mathrm{g} / \mathrm{m}^{3}$ (Fig $2 \mathrm{~b}$ ). A very bad smoke day (average smoke $\mathrm{PM}_{2.5}$ concentration of $100 \mu \mathrm{g} / \mathrm{m}^{3}$ ) is associated with a roughly 0.2 standard deviation decline in sentiment relative to the overall sample standard deviation. For context, the average difference between tweet sentiment on Wednesdays and Saturdays (respectively, the lowest- and highest-sentiment days of the week in our data) is about 0.007 in our data; one day of very bad smoke $\left(100 \mu \mathrm{g} / \mathrm{m}^{3}\right)$ is thus about 3 times worse in sentiment terms than replacing an average Saturday with an average Wednesday.

Negative effects of smoke on sentiment could occur through a variety of channels, including from fear or anxiety about proximate fires themselves or about what the fires represent (e.g. a changing climate), from unhappiness due to disruption in normal activities (e.g school closure or inability to recreate), or from anticipation or experience of negative health impacts. While we cannot distinguish the latter channels in our data, effects of smoke on sentiment are not driven by proximity to active wildfire, and are robust to temperature and rainfall controls and to alternate fixed effects (Table S5).

Exposure to smoke $\mathrm{PM}_{2.5}$ increases search activity related to protective behavior. Searches for technologies known to help limit exposure, including "air filter", "air purifier", "smoke mask", and "PurpleAir", all increase on days in which smoke exposure is higher (Fig 2c, Table S6). Some search queries in Spanish ("purificador de aire") respond similarly, although others ("filtro de aire") do not (Table S6).

Finally, smoke $\mathrm{PM}_{2.5}$ exposure on average causes more people to not leave their home, with immediate increases at low levels of exposure that flatten off at high levels (Fig 2d). A day of smoke exposure above $50 \mu \mathrm{g} / \mathrm{m}^{3}$ leads to a roughly 3 percentage point increase in the proportion of people fully at home, which corresponds to about a $10 \%$ increase above the mean. Smoke $\mathrm{PM}_{2.5}$ exposure has a limited effect on the proportion of people fully away from their home at low expo- 
sure levels, but an increasing effect at higher exposure levels (Fig S4). Both results can be interpreted as protective behavior: during heavy smoke days, many individuals shelter in their homes, and some leave the area when exposure gets severe. Both results are robust to controls and are not driven by proximity to active fires; the effect on percent of people at home is less robust to the addition of more stringent time controls (Table S7).

Exposure and response heterogeneity Individuals are likely to respond to environmental exposures in different ways, either because their personal exposure varies or because, for a given exposure, their knowledge of that exposure or their willingness or ability to respond to it could differ. We explore heterogeneous exposures and responses to wildfire smoke as a function of socioeconomic status (as measured by locality-specific median household income) and variation in average previous exposure to wildfire or other $\mathrm{PM}_{2.5}$. Previous literature suggests both could moderate behavioral responses to environmental stress through a variety of mechanisms, including through differential access to information about exposure risk or differential ability, motivation, or knowledge of how to take protective action $(8,20,42)$.

Consistent with earlier work (10), but in stark contrast to strong socioeconomic and ethnic/racial gradients in exposure to other key pollutants in the US $(43,44)$, we find that exposure to both average and acute smoke $\mathrm{PM}_{2.5}$ is largely uncorrelated with income in the US (Fig S3). We also find no differences in salience of smoke exposure between lower- and higher-income counties, with similar responses of search query activity to a day of heavy smoke across income levels (Fig 3a)

Other behavioral measures show strong income gradients. For sentiment, wealthier counties respond much more negatively to a heavy smoke day than less wealthy counties (Fig 3b). This finding is not driven by average differences in sentiment between more and less wealthy counties, by higher overall variation in sentiment in wealthier versus poorer counties (temporal variation in sentiment is lower in wealthy counties than in less-wealthy counties in our sample), or by differences in average exposure to smoke $\mathrm{PM}_{2.5}$ or other sources of $\mathrm{PM}_{2.5}$ (Table S9).

Search activity related to protective behavior is also substantially higher in wealthier counties (Fig 3c), and not statistically different from zero in roughly the bottom third of the county income distribution. Finally, populations in wealthier counties are also substantially more likely to remain fully at home during a day of heavy wildfire smoke exposure than lower-income populations (Fig 3d). Results are robust to more or less restrictive time controls (Fig S5a). We find no meaningful difference across income groups in the proportion of individuals fully away from their house during days of heavy smoke exposure (Fig S5b). 
Why do wealthier locations respond differentially to smoke exposure? Measured differences do not appear to reflect differences in exposure information or in overall internet activity, given the consistent response of air-quality related searches across income groups. Rather, responses are consistent with lower incomes constraining choice sets and behaviors, including less flexibility in working from home, fewer resources with which to consider purchasing protective technology, and - regarding the sentiment results - having other more pressing matters to worry about.

We find that behavioral measures are also affected by previous experience with smoke and with other $\mathrm{PM}_{2.5}$ sources. An additional smoke day was less salient in locations with higher previous exposure to smoke $\mathrm{PM}_{2.5}$, and locations with higher average $\mathrm{PM}_{2.5}$ exposure prior to our study period showed smaller declines in sentiment during an additional high smoke day and fewer searches related to health-protective behavior, but were more likely to stay at home when smoke $\mathrm{PM}_{2.5}$ was high (Tables S8-S9). Results are consistent with individuals adapting their behavior and beliefs based on repeated exposure, e.g. through investments in health-protective technologies.

Smoke $\mathbf{P M}_{2.5}$ infiltration into indoor environments We find that Census tracts with PurpleAir monitors tend to be wealthier on average than tracts without monitors (Fig S6), a finding consistent with other analyses (45) and with the income-differentiated search activity for "PurpleAir" and related health-protective technologies found above. Nevertheless, the average income of locations owning indoor monitors varies by 10x across locations, enabling an exploration of the role of income and other demographic factors in shaping exposures among a population with identical access to information on their exposures.

Using a pooled model we estimate that a $1-\mu \mathrm{g} / \mathrm{m}^{3}$ increase in outdoor $\mathrm{PM}_{2.5}$ is associated with an 0.15 (95\% CI: $0.135-0.153) \mu g / m^{3}$ increase in indoor $\mathrm{PM}_{2.5}$ over the next six hours. Estimates are robust to alternate regression models and alternate corrections to the monitor data (Fig S7) and are comparable in magnitude to recently published estimates (40). Estimated infiltration is substantially lower during periods of very high outdoor $\mathrm{PM}_{2.5}$ (Fig 4a). However, for a given outdoor $\mathrm{PM}_{2.5}$ level, infiltration is higher when at least some of the $\mathrm{PM}_{2.5}$ is from smoke. Earlier findings of lower infiltration on smoke days (33) were likely capturing the effect of overall high $\mathrm{PM}_{2.5}$ rather than the effect of smoke-derived $\mathrm{PM}_{2.5}$ specifically.

Consistent with our other behavioral measures, declining infiltration at high outdoor $\mathrm{PM}_{2.5}$ levels suggests that salient ambient exposures induce behavioral responses, which could include closing windows or doors and/or using mechanical filtration. However, in contrast with our other behavioral measures, we find only a modest relationship between neighborhood average income and infiltration, with households in much wealthier Census blocks experiencing only slightly lower 
average infiltration than households in areas with one quarter the average income regardless of whether the $\mathrm{PM}_{2.5}$ was smoke-derived (Fig 4b).

To further explore predictors of infiltration, we estimate infiltration separately for each of the 1,520 indoor monitors in our dataset, match each monitor to a wide range of house- and neighborhoodspecific socioeconomic, demographic, environmental, and housing covariates, and fit flexible machine learning models relating infiltration to these covariates (Methods). Consistent with other work (40), we find many-fold differences in household-specific infiltration rates (mean = 0.19 , sd = 0.16; see Fig 4c), and confirm using a Bayesian hierarchical model that this variation is largely due to "true" underlying variation between households rather than to sampling noise in household-level estimates (Fig S8a). Estimates are only modestly correlated with traditional indoor/outdoor ratio estimates (Fig S9), perhaps due to the difficulty in accounting for indoor sources of emissions or diurnal behavioral patterns in the traditional I/O approach (Methods).

While racial/ethnic, socioeconomic, environmental, and housing variables are associated with infiltration on held-out data, their individual explanatory power is very modest, and our rich set of predictors and flexible models are surprisingly poor predictors of overall variation in infiltration, explaining only $\sim 5 \%$ of variation across indoor monitors in our data (Fig S8b-c). This lack of predictive ability of socioeconomic factors is also apparent on individual smoke days, where even among relatively socioeconomically advantaged households, very similar outdoor $\mathrm{PM}_{2.5}$ concentrations during a given smoke day are associated with widely varying indoor $\mathrm{PM}_{2.5}$ concentrations (Fig S10).

To further investigate the differential influence of behavior versus housing characteristics (and associated socioeconomic factors), we re-estimated infiltration for individual households during periods when windows were likely to be closed and indoor filtration not running (Methods). While average infiltration during these periods was relatively similar to infiltration during all periods (Fig S8d), infiltration varies much more strongly with both income (Fig S8e) and housing age (S8f) under these conditions. Taken together, and consistent with previous smaller-scale work (39), our results indicate that the poor explanatory power of socioeconomic and housing characteristics is not driven by poor measurement of these characteristics but by the dominant effect of idiosyncratic household-specific behaviors that are not correlated with these characteristics.

Finally, using indoor monitors across the Bay Area and data prior to Aug 2020, we divide monitors into low (bottom quartile) and high (top quartile) infiltration groups (Fig 4d) and study outdoor and indoor $\mathrm{PM}_{2.5}$ levels across these groups during the extreme wildfire smoke event the area experienced in Aug-Sep 2020. High and low infiltration households experienced nearly iden- 
tical daily outdoor concentrations during the many-week event (Fig 4e), but these ambient levels led to starkly different indoor concentrations. On the worst smoke days, daily average indoor concentrations across all high infiltration homes exceeded $65 \mu \mathrm{g} / \mathrm{m}^{3}$ and in some houses it exceeded $100 \mu \mathrm{g} / \mathrm{m}^{3}$, well above the World Health Organization 24-hour $\mathrm{PM}_{2.5}$ exposure guideline of $15 \mu \mathrm{g} / \mathrm{m}^{3}$. Low infiltration households were on average able to maintain indoor $\mathrm{PM}_{2.5}$ concentrations near $5 \mu \mathrm{g} / \mathrm{m}^{3}$. Across the duration of the smoke event, daily mean indoor concentrations were on average 3.5x higher in the highest quartile versus the lowest quartile of infiltration households. Differences were even larger when looking across all Bay Area monitors: households with average outdoor $\mathrm{PM}_{2.5}$ levels within $5 \mu \mathrm{g} / \mathrm{m}^{3}$ of each other experienced $>20 \mathrm{x}$ differences in average indoor $\mathrm{PM}_{2.5}$ concentrations during the smoke event (Fig S11).

\section{Discussion}

A growing literature documents the large and often disparate impacts of wildfire smoke on a range of health outcomes $(14-18,21,22)$. Our results show how novel sensor data can provide policy-relevant insight into why the magnitude and incidence of these impacts might vary. Multiple lines of evidence indicate that awareness of smoke concentrations does not appear to be a primary constraint on individual behavior in the face of wildfire smoke exposure: even small increases in ambient exposure cause individuals to seek air quality information, become unhappier, and stay in their homes. But while awareness appears to be broadly shared, it does not lead to adequate health protection. Even among populations that own indoor monitors and who thus have access to accurate, real-time measures of their indoor concentrations, information is not enough to limit dangerous indoor exposures to these pollutants. This suggests policies that target information provision about smoke are insufficient, and perhaps not central, to enabling protective behavior.

Socioeconomic status is not correlated with outdoor smoke levels but does appear to mediate behavioral responses to such pollution. Wealthier households in our sample can more easily stay home, are more likely to seek information on protective technology, and are more likely to own indoor pollution monitors. Such differential behavior is consistent with a broader literature that shows how socioeconomic status constrains households' abilities to invest in environmental quality and health protection $(8,46)$. Yet, at least among our sample of monitor-owning households, income is only weakly correlated with the infiltration of ambient smoke into indoor environments, and we observe many households in wealthy neighborhoods experiencing exceedingly high levels of indoor smoke exposure. 
Our results suggest this is likely because present infiltration rates are dominated by actions like opening windows and doors, not housing materials or quality that might be reflected in prices. Infiltration patterns thus point to the importance of behavior that remains unobserved, a fact which is both encouraging and troubling. If simple but difficult-to-observe behaviors such as closing windows and doors explain the vast majority of variation in smoke infiltration, then reducing infiltration at population scale could be much easier in theory than if infiltration was largely determined by income or housing quality, as changing these latter factors requires addressing deeper societal problems of inequality and structural racism.

On the other hand, current policy approaches to addressing smoke exposure focus on behavioral recommendations to stay at home and close windows and doors (24), but these policies alone are difficult to comply with and may still be inadequate: many households' indoor environments remain highly exposed, and our mobility results suggest adherence might be difficult for lowerincome households. If such behaviors are indeed hard to adopt, then the policy approach of promoting private provision of protection could be biased against disadvantaged groups. This policy approach also stands in stark contrast to the approach of public provision of protection used for other sources of $\mathrm{PM}_{2.5}$, which has sought to reduce emissions of pollutants at their source and which has successfully reduced overall ambient exposure inequalities (47). Further understanding the variation and causes of the behaviors that can protect indoor environments will be key to designing policy that can both lower indoor concentrations and not disadvantage certain groups. 

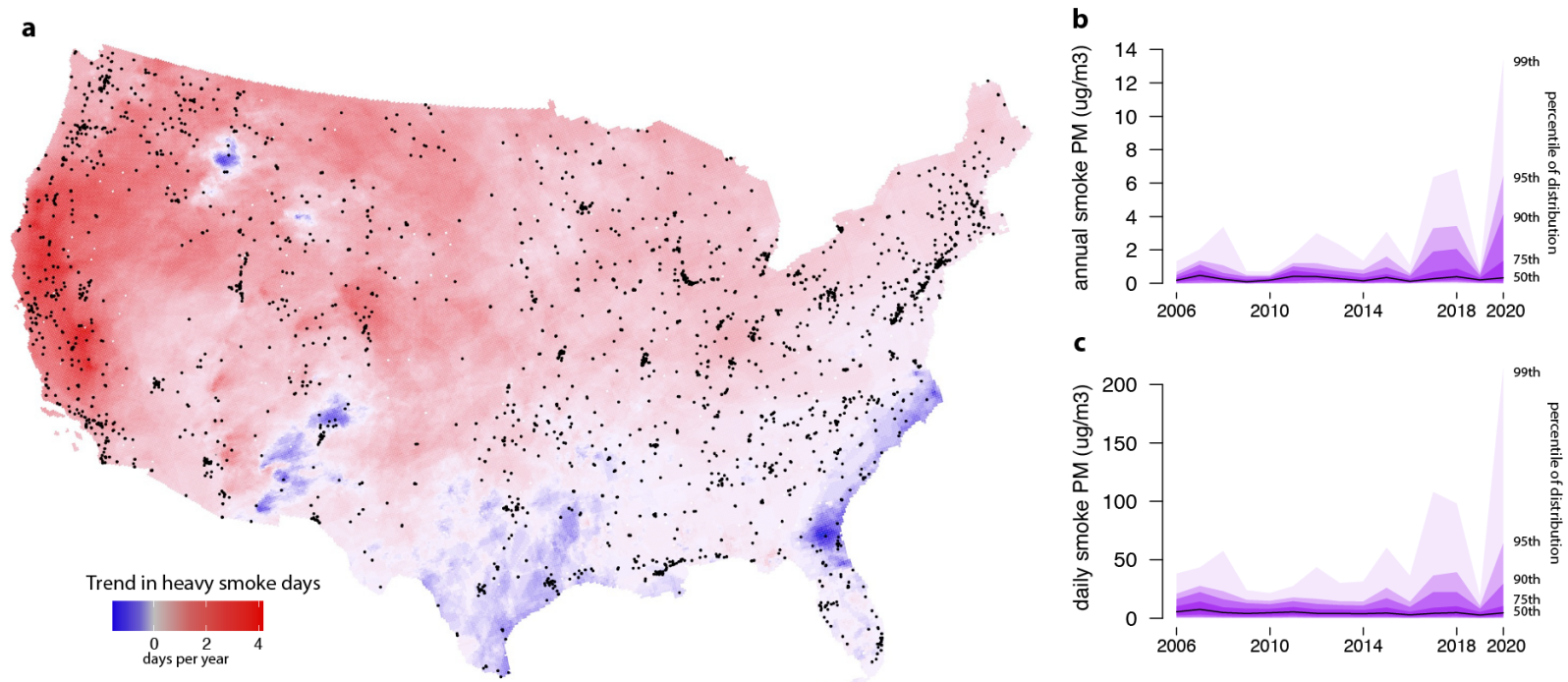

Figure 1: Trends in smoke exposure across the US. a Measurements from satellites indicate rapidly growing exposure to "heavy" smoke plumes across much of the US. Estimates are shown on a 10km grid and indicate the estimated annual increase between 2011 and 2020 in the number of smoke plumes that NOAA analysts designate as "heavy", their densest plume classification. Dots indicate EPA ground-based pollution monitors. b Distribution of estimated annual average smoke $\mathrm{PM}_{2.5}$ across EPA ground monitors reporting in each year. c Distribution of estimated daily smoke $\mathrm{PM}_{2.5}$ across the same monitors. Recent increases in extreme annual exposure are being driven by increases in extreme daily exposure. 

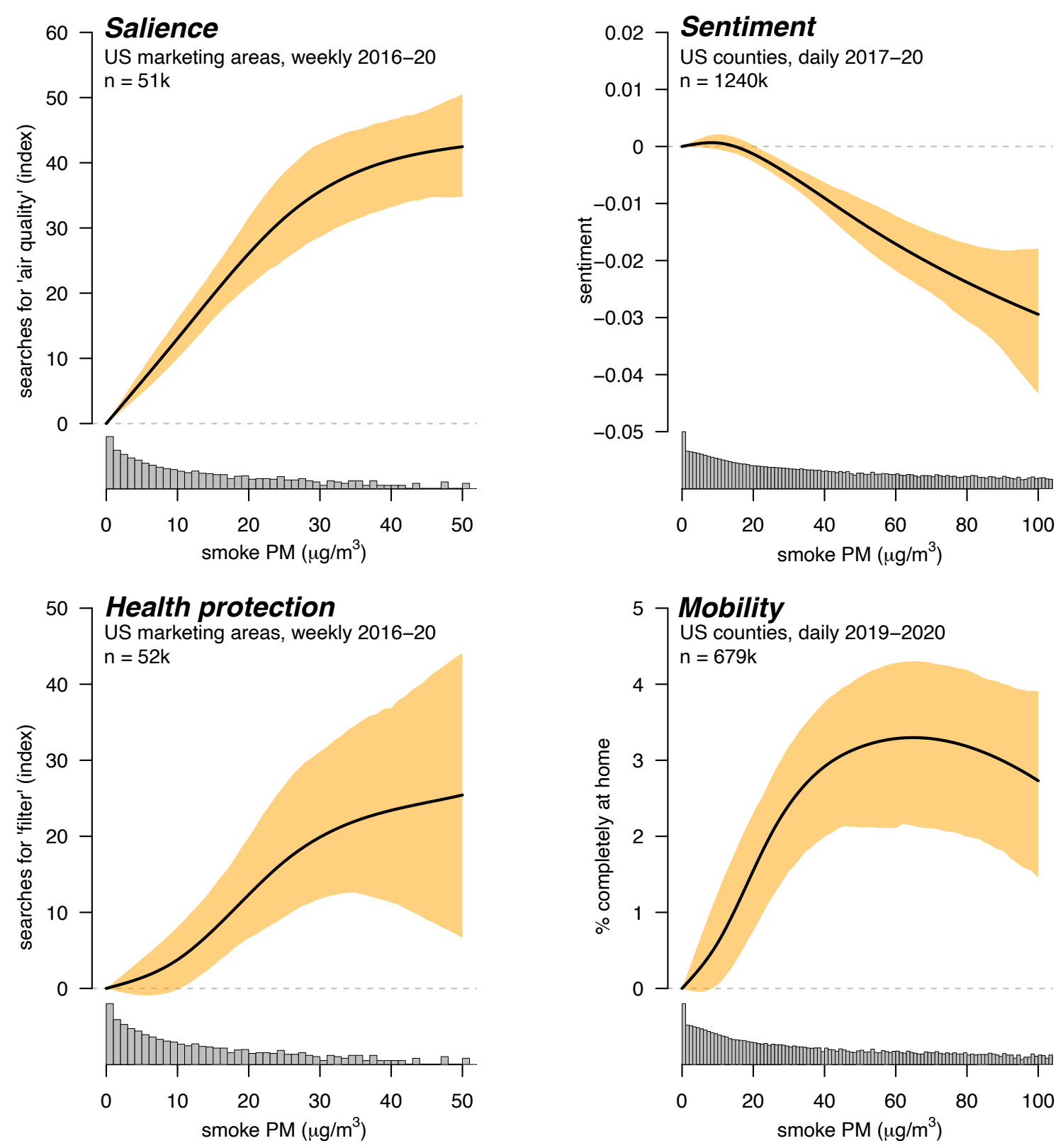

Figure 2: Behavioral responses to wildfire smoke exposure. a Searches for 'air quality' on Google at the US designated marketing area by week level, 2016-2020. Search index is normalized such that zero indicates no searches and 100 indicates the maximum number of weekly searches over the period. b Average sentiment in Twitter updates ('tweets') at the county-day level, 2017-2020. c Searches for 'air filter' on Google at the US marketing area by week level, 2016-2020. Search index normalized as in (a). d Percent of mobile phones estimated to be completely at home on a given day at the US county level, 2019-2020. Lines are spline fits conditional on fixed effects, with shaded areas showing bootstrapped 95\% confidence intervals. Number of observations in each regression is shown in upper left corner of each panel. Histograms at the bottom show the $\log$ distribution of smoke $\mathrm{PM}_{2.5}$ exposure in each sample. 

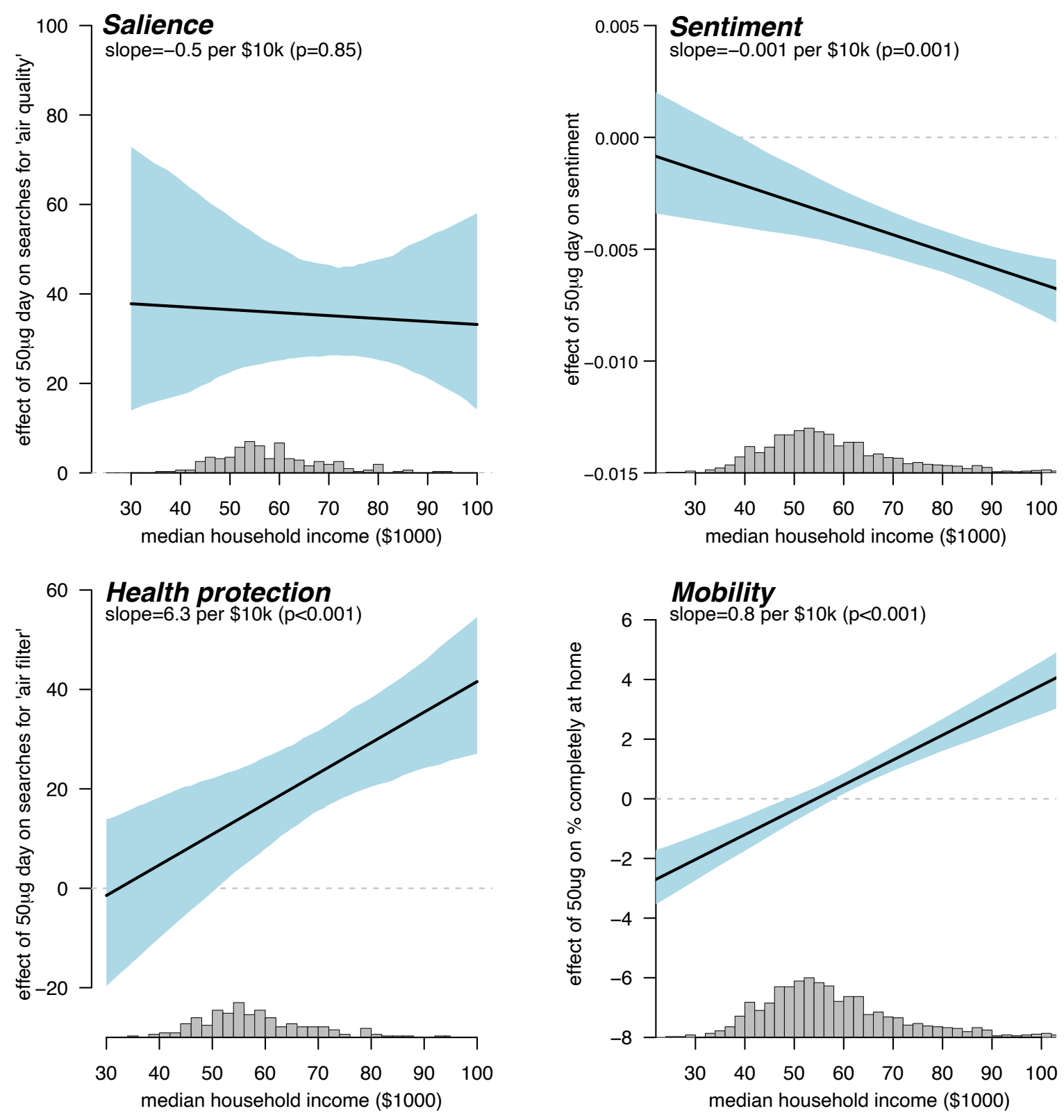

Figure 3: Smoke salience does not differ, but other responses do differ, among more and less wealthy populations. a The effect of a heavy smoke exposure $\left(50 \mu \mathrm{g}\right.$ smoke $\mathrm{PM}_{2.5}$ on that day) on searches for 'air quality' on Google do not differ by income. b A heavy smoke week has a stronger negative effect on sentiment among wealthy populations. $\mathbf{c}$ Wealthier populations are substantially more likely to search for 'air filter' on Google during a heavy smoke day. d Wealthier populations are more likely to be completely at home during a week of heavy smoke. Lines show the marginal effect of a $50 \mu \mathrm{g}$ smoke day on the outcome at different income levels, with shaded areas showing bootstrapped $95 \%$ confidence intervals. The slope of each line is shown in upper left, and the histograms at bottom show the distribution of county incomes in each sample. Sample in each panel is same as corresponding panels in Fig 2. 

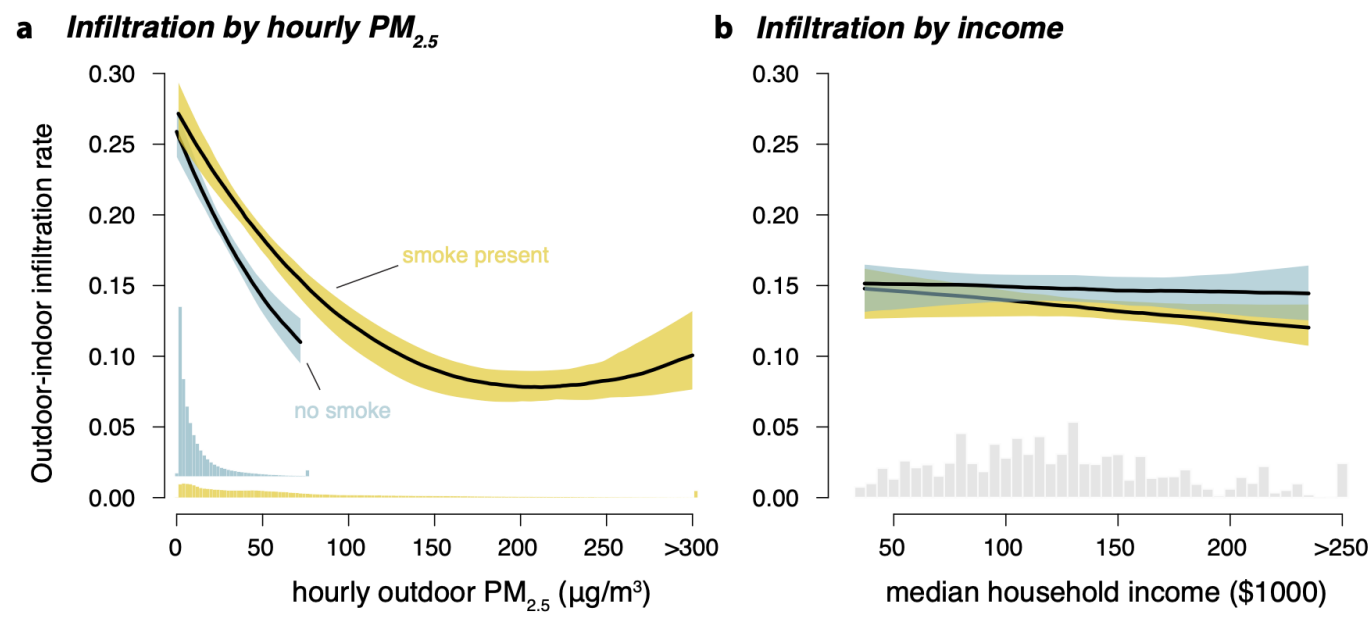

C Infiltration across sample households
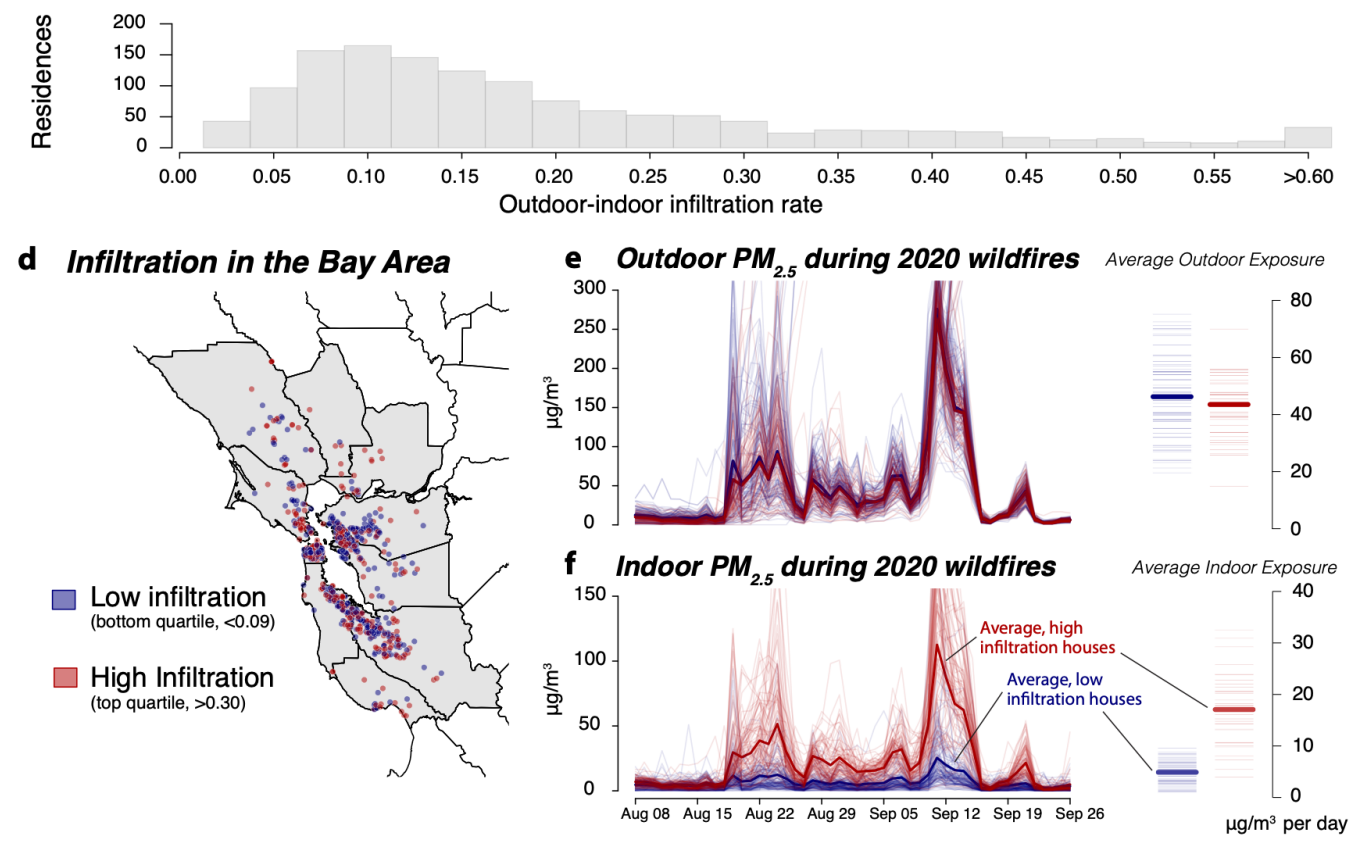

Figure 4: Infiltration rates decline strongly with outdoor $\mathbf{P} \mathbf{M}_{2.5}$ concentration during both fire and non-fire periods, but not with income, and differ greatly across households resulting in extreme differences in indoor exposure during wildfires. a Infiltration rates, measured as the integrated hourly change in indoor $\mathrm{PM}_{2.5}$ per unit increase in outdoor $\mathrm{PM}_{2.5}$, are lower during periods of high outdoor $\mathrm{PM}_{2.5}$ regardless of whether $\mathrm{PM}_{2.5}$ comes from smoke or other sources. b Infiltration rates are only weakly correlated with census block median income. c The distribution of infiltration rates estimated separately for each household in the sample is wide. d Residents from the highest and lowest infiltration quartiles in the Bay Area are mapped and colored according to group, showing the groups are geographically intermixed. e Daily outdoor $\mathrm{PM}_{2.5}$ concentrations during unprecendented Aug/Sep 2020 wildfire smoke event were highly similar between high and low infiltration households. Transparent lines show concentrations at each residence and thick lines show the averages within each group. The left panel shows daily mean concentrations and the right panel shows averages across all days during the event. f Same as e but for indoor $\mathrm{PM}_{2.5}$ concentrations, showing very large $(>100 \mu \mathrm{g})$ daily differences during peak outdoor concentrations (left) and 3.5x differences in average indoor exposure between high and low infiltration groups (right). 


\section{Methods}

Estimating ground $\mathbf{P M}_{2.5}$ concentrations from smoke We develop a generic, tractable method for estimating ground $\mathrm{PM}_{2.5}$ attributable to smoke at the daily level. The method requires a credible estimate of whether there is smoke in the air on a given day, and a daily time series of $\mathrm{PM}_{2.5}$ from which location- and period-specific anomalies can be constructed. In principle, any available (accurate) daily $\mathrm{PM}_{2.5}$ estimates could be used, including promising recent machine-learningbased efforts at generating high-resolution gridded time series of $\mathrm{PM}_{2.5}$ concentrations $(48,49)$. However, existing gridded data are not available for recent years, and so we instead use stationbased daily $\mathrm{PM}_{2.5}$ measures from the network of thousands of EPA stations across the continental US (Fig 1a).

To construct our daily measures of smoke $\mathrm{PM}_{2.5}$, we define $P M_{i d m y}$ as the $\mathrm{PM}_{2.5}$ concentration recorded by the EPA monitor at location i on day d, month $\mathrm{m}$, and year $\mathrm{y}$. From this time series we construct location- and month-specific anomalies $P M_{a n o m}{ }_{i d m y}=P M_{i d m y}-P \bar{M}_{i m y}$, where $P \bar{M}_{i m y}$ is monthly median $\mathrm{PM}_{2.5}$ on non-smoke days at that location, and where median is defined over the three years surrounding the year of interest. So for example, a $\mathrm{PM}_{2.5}$ anomaly for the Redwood City CA EPA station on Jan 10, 2019 is calculated as the value on Jan 10, 2019 minus the median $\mathrm{PM}_{2.5}$ value on all January days in 2018, 2019, and 2020 in Redwood City when smoke was not overhead. Our measure of whether smoke was overhead, plume idmy $_{\text {, is derived }}$ from the National Oceanic and Atmospheric Administration (NOAA) Hazard Mapping System (HMS) satellite estimates of smoke plume boundaries. We define plume $_{i d m y}=1$ if there was a smoke plume of any thickness over location $i$ during any time on day $d$, and zero otherwise. Finally, from these data we can construct $S m o k e P M_{i d m y}=P_{M a n o m}$ idmt $*$ plume $_{i d m y}$. SmokePMidmy will thus equal zero when there is no plume overhead, and will equal the anomaly value when there is smoke overhead. Our approach thus provides a continuous measure of smoke exposure intensity.

Our approach is similar to recent work (50) using interpolated station data and plumes to estimate smoke $\mathrm{PM}_{2.5}$. However, given the high spatial variation in smoke exposure and often large distance between EPA stations, we chose not to interpolate EPA stations. Given the secular decline in background $\mathrm{PM}_{2.5}$ across the country, we also define anomalies relative to a moving (3-year) monthly median rather than a fixed seasonal mean.

Measuring salience and health-protective behavior We measure salience and health-protective behavior using public search query data from Google Trends. Data are accessed using the $\mathrm{R}$ package gtrendsR, and are provided as location-, term- and period-normalized indices ranging 
from 0 to 100, where 0 is the lowest search volume for that term in that location during the chosen period, and 100 is the highest search volume. Data are available at the "Designated Market Area" level (referred to as "Metro" areas by Google Trends), which are geographic regions encompassing television media markets as defined by Nielsen.

We study searches in both English and Spanish, which together are the primary languages spoken by $92 \%$ of US households (51). We use weekly data on designated market areas, the native spatial resolution of the public Trends data, between Jan 2016 and Dec 2020, and analyze data on terms related to smoke exposure (including "air quality", "smoke", and "wildfire smoke").

Measuring sentiment We measure online sentiment for a county-day using the text of Twitter posts ("tweets") created in that county on that day. Specifically, we collect nearly all of the geolocated tweets for the continental United States between December 2016 and February 2021 through the Twitter Streaming API. To compute sentiment for each tweet, we apply the VADER sentiment analysis model (28), a natural language processing algorithm tuned specifically for estimating sentiment from online language. We take the average of the "compound" scores (ranging between -1 and 1) computed by VADER for all tweets in a county-day as our measurement of sentiment. Our approach builds on the computation of expressed sentiment described in ref (26). Readers may refer to that article for additional details on the general approach to collecting and processing tweets for use in empirical analysis. On average, mean sentiment for a county-day is 0.17 , computed from 455.4 tweets.

Measuring mobility We assembled a daily dataset of mobility measures at the county-level collected between Jan 2019-Dec 2020, the period over which mobility data were generously made available to researchers by Safegraph. These data measure the aggregate activity of anonymized device signals, or "pings", at the census block group-level. Signals are collected from smartphones, not all cell phones. We focus on two measures constructed from these anonymized signals: percentage of individuals completely at home on that day, and percentage of individuals completely away from home on that day. We construct the completely away from home variable by counting the percentage of devices on a given day that were not observed in their respective home location. Safegraph assigns a home location to each device based on their mobility pattern observed over the previous six weeks. We aggregate these data to the county-day level by taking means weighted by the number of devices in each census block. Data processing details are discussed further in refs (52-54).

Measuring distance to fire To distinguish the effects of exposure to wildfire smoke from potentially correlated effects of being near an active wildfire, we develop daily measures of proximity to active wildfires and control for them in the regression analysis. Our measures of active fires 
are derived from NOAA HMS fire points. Because these fire detections often indicate very small and isolated fires that likely do not represent threatening wildfires, and large active fires typically have many nearby fire detections, we build on earlier work (10) and denote active fires by constructing "fire clusters", or locations with multiple active nearby fire points. To do this, we buffer each HMS fire point by $3 \mathrm{~km}$ square, and take the union of existing overlapping squares over a given day and previous three days. We then define an active fire as a location where this union is $>10 \mathrm{~km}^{2}$. This does not mean $10 \mathrm{~km}^{2}$ are burning, but within that $10 \mathrm{~km}^{2}$ there are multiple fire points over a three day period, representing an active and potentially growing fire. We then compute "distance to fire" as the distance from a geography centroid (e.g. county) to the nearest active fire, setting this distance to 0 if the active fire cluster is inside the county on that day.

Estimating ambient smoke impact We combine the above behavioral measures with our smoke $\mathrm{PM}_{2.5}$ estimates and analyze their correspondence using panel fixed effects estimators, with the goal of isolating the impact of variation in smoke exposure from other time-invariant and timevarying factors that could be correlated with both smoke exposure and outcomes. Specifically, we estimate econometric models of the form:

$$
y_{i s d m y}=f\left(\text { SmokePM } M_{i s m y}\right)+Z_{i s m y}+\alpha_{i m}+\eta_{d}+\varepsilon_{i s d m y}
$$

where $y_{i s d m t}$ is outcome of interest in unit $i$, state $s$, day $d$, month $m$, and year $y$, SmokePMismy is our smoke $\mathrm{PM}_{2.5}$ measure on the same day and location, and $Z_{\text {ismy }}$ are additional time-varying controls. Our preferred model includes location-by-month FE to account for local seasonality in either outcomes or exposures (i.e. one intercept for each of the twelve months in Santa Clara County, CA), day-of-sample FE (e.g. a dummy for Jan 1, another for Jan 2 2016, etc) to account for common trends or shocks to outcomes or exposures on a given day. Our date FE implicitly also account for any average differences in outcomes between weekends and weekdays. We estimate $f()$ using either linear models or more flexible cubic splines to capture potential nonlinearities. In all analyses using search query data, Twitter data, or mobility data, smoke $\mathrm{PM}_{2.5}$ is measured using EPA station data, as described above.

In these models, the effect of smoke exposure on outcome $y$ is estimated by relating, e.g., outcomes in Santa Clara County on Aug 302020 versus Sep 12020 to differences in smoke exposure on those days, after accounting for any common difference across counties in exposure or outcomes between the two days, and any average differences in smoke exposure or outcomes in August vs September in Santa Clara County. A confounding variable would have to be a local time-trending unobservable correlated with both smoke exposure and the outcome. Possible candidates include weather variables and the presence of an active wildfire nearby, and we addi- 
tionally control for these variables $\left(Z_{i s m y}\right.$ in Equation 1) in robustness tests, or split the sample between locations nearby and further from an active wildfire.

Another potential threat to identification is the COVID epidemic, which near the end of our sample period had demonstrated effects on mobility (55) and sentiment (56), and likely enhanced awareness about the importance of air filtration (57); 2020 was also a year of severe smoke exposure throughout much of the US West. Because we exploit daily variation in smoke exposure over time at particular locations, and because such variation depends largely on stochastic factors such as exactly where fires ignite and which way the wind is blowing, we believe daily variation in COVID outcomes or behaviors are unlikely correlated with wildfire smoke exposures. However, to further address this confounding risk, we test robustness to even more stringent time controls, including county by month of sample FE, and state by day of sample FE; these further account for any state-specific differences or trends in COVID severity and/or policy intervention that happened to coincide with wildfire risk. We note that any changes in our observed behavioral outcomes due to wildfire-specific effects on health outcomes, including wildfire's potential effects on COVID itself (17), are not confounding and would constitute part of the overall "effect" that we wish to understand.

To study whether the effects of smoke on outcomes vary across locations, we interact smoke exposure with time-invariant covariates of interest:

$$
y_{i s d m y}=\beta_{1} \text { SmokePM } M_{i s m y}+\beta_{2} \text { SmokePM } M_{i s m y} * X_{i}+Z_{i s m y}+\alpha_{i m}+\eta_{d}+\varepsilon_{i s d m y}
$$

where $X_{i}$ in our analysis include median household income, average previous exposure to $\mathrm{PM}_{2.5}$, and average smoke $\mathrm{PM}_{2.5}$ exposure, either included individually or jointly.

Measuring indoor and outdoor household $\mathbf{P M}_{2.5}$ using Purple Air To estimate household infiltration of outdoor $\mathrm{PM}_{2.5}$ into indoor environments, we utilize data collected by low-cost Purple Air monitors measuring indoor and outdoor $\mathrm{PM}_{2.5}$ concentrations. Raw 10-minute observations were downloaded for all available indoor and outdoor Purple Air monitors in the contiguous United States and then cleaned according to the procedures developed by recent studies $(38,40)$ in order to produce hourly indoor and outdoor $\mathrm{PM}_{2.5}$ concentrations. Namely, for each monitor, we download 10-minute observations from the earliest available date through the end of 2020 or the last available date, whichever is later.

Following existing literature, we use multiple approaches for estimating $\mathrm{PM}_{2.5}$ concentrations from Purple Air data. In a "particle count" approach, we derive 10-min $\mathrm{PM}_{2.5}$ concentrations based on the reported particle counts reported by each sensor (i.e., for dual sensor monitors two 
different $\mathrm{PM}_{2.5}$ concentrations were derived for each 10-min period, one for each sensor). $\mathrm{PM}_{2.5}$ concentrations were derived from particle counts using equations 1 and 2 from Bi et al 2021 (38). For the other approaches that relied directly on Purple Air's reported concentrations we record the 10-min average $\mathrm{PM}_{2.5}$ reported in the cf_1 channel. We choose the cf_1 channel rather than the cf_atm channel because previous work has shown it correlates better with concentrations measured by EPA monitors (58). Furthermore, because Purple Air previously had the cf_1 and cf_atm columns incorrectly labeled in their data, we use the updated labels for each channel to ensure we have correctly identified the cf_1 observation. Then, for all approaches the following steps were used to go from the 10-minute Purple Air $\mathrm{PM}_{2.5}$ concentrations to the final cleaned hourly data used in the analysis:

1. For monitors with A and B sensors, we compare the two sensors and set any 10-min observation to NA if the difference between sensors exceeds a certain threshold. Specifically, when $\mathrm{PM}_{2.5} \leq 100 \mu \mathrm{g} / \mathrm{m}^{3}$ we set the observation to NA if the A and B sensors differ by more than $10 \mu \mathrm{g} / \mathrm{m}^{3}$ and when $\mathrm{PM}_{2.5}>100$ we set the observation to NA if the A and $\mathrm{B}$ channels differ by more than $10 \%$. We then take the average across the two sensors. Observations where either sensor has a missing value are set to missing.

2. Next, we examine all adjacent three period runs to see if there are 'spikes' defined as an increase and then immediate decrease in the subsequent period or a decrease and then subsequent increase in the next period. If the mirror jumps in subsequent periods from $t-1$ to $t$ and from $t$ to $t+1$ are both over 100 then we set observation in time $t$ to NA.

3. To get hourly observations we take the 60 -min mean, setting the hourly average to NA if any 10 -min period was missing.

This process resulted in two separate measures of $\mathrm{PM}_{2.5}$ concentrations for each monitor, one based on particle counts and one based on Purple Air's reported $\mathrm{PM}_{2.5}$ concentrations. We then derived a third measure of $\mathrm{PM}_{2.5}$ concentrations by using the Purple Air reported concentrations (i.e., not those derived from the particle counts) and applying the correction developed in Barkjohn et al 2021 (58) to improve agreement between Purple Air $\mathrm{PM}_{2.5}$ concentrations and EPA reference monitor concentrations.

Hourly ambient exposures were estimated at each indoor monitor site by first identifying all outdoor monitors within $5 \mathrm{~km}$ and then taking the inverse distance weighted average of hourly $\mathrm{PM}_{2.5}$ concentration across the (up to) 10 nearest monitors. Monitors with less than 720 non-missing 
hourly indoor and outdoor $\mathrm{PM}_{2.5}$ measurements (i.e., 30 days of hourly data) were excluded from the analysis.

Finally, indoor Purple Air monitors are deployed in many different types of buildings. We used a combination of information from monitor labels and manually checking of geolocations to determine which buildings with indoor Purple Air monitors were single family residences (SFR). All other types of buildings were removed from the sample. In total, there were 1,520 monitors reporting in our sample of single family residences.

In order to validate the three versions of Purple Air derived $\mathrm{PM}_{2.5}$ concentrations we identify the nearest outdoor Purple Air monitor to each available EPA monitor that reports hourly data and we estimate the following regression:

$$
P M_{i h m}^{E P A}=\beta P M_{j h m}^{P A}+\alpha_{i}+\theta_{h}+\eta_{m}+\varepsilon_{i h m}
$$

where $P M_{i h m}^{E P A}$ is the EPA reported hourly $\mathrm{PM}_{2.5}$ concentration for monitor $i$ in hour-of-day $h$ and for month-of-sample $m$ and $P M_{j h m}^{P A}$ is one of the three versions of hourly PM2.5 concentration estimated at the nearest Purple Air monitor. Our infiltration measure derived from these data is a regression based estimate that relates anomalies (i.e. deviations from averages along several dimensions) in indoor $\mathrm{PM}_{2.5}$ to anomalies in outdoor $\mathrm{PM}_{2.5}$. Therefore when evaluating performance of the Purple Air $\mathrm{PM}_{2.5}$ measures with respect to EPA monitors, we include monitor, hour-of-day, and month-of-sample fixed effects in the comparison regression.

Results from estimation of the EPA monitor comparison regressions are shown in Table S1. Anomalies in all three versions of Purple Air $\mathrm{PM}_{2.5}$ measures are strongly correlated with anomalies in hourly measures from nearby EPA monitors with $\mathrm{R}^{2}$ values in each case $>0.65$. The uncorrected Purple Air cf_1 $\mathrm{PM}_{2.5}$ measures overestimate EPA $\mathrm{PM}_{2.5}$ and explain the smallest share of the variation in anomalies. Unsurprisingly, given the Barkjohn et al correction was designed specifically to calibrate Purple Air $\mathrm{PM}_{2.5}$ to EPA $\mathrm{PM}_{2.5}$ levels, it is the best of the three measures at predicting the level of EPA monitor $\mathrm{PM}_{2.5}$. However, while the particle count derived $\mathrm{PM}_{2.5}$ concentrations underestimate EPA monitor concentrations, they explains the largest share of the variation $\left(\mathrm{R}^{2}=0.72\right)$. This is consistent with a recent study on Purple Air monitor $\mathrm{PM}_{2.5}$ calibration that found $\mathrm{PM}_{2.5}$ concentrations derived from Purple Air particle counts best explained EPA measured $\mathrm{PM}_{2.5}$ anomalies both overall and specifically during wildfire events (59). In light of these findings, we follow several recent publications $(38,40,59)$ and present our main infiltration results with $\mathrm{PM}_{2.5}$ concentrations derived from Purple Air particle counts. However, we also 
present infiltration estimates for all three versions of $\mathrm{PM}_{2.5}$ concentrations (in each case using the same measure for indoor and outdoor concentrations) and find estimates are highly correlated across choice of measure (Fig S7).

Estimating infiltration rates In order to estimate the average indoor infiltration rate, which we define as the increase in indoor $\mathrm{PM}_{2.5}$ concentration per unit increase in local outdoor $\mathrm{PM}_{2.5}$ concentration (i.e., $\partial$ Indoor $P M_{2.5} / \partial$ Outdoor $P M_{2.5}$ ), we estimate a regression at the monitorhour level. Namely, for each residence $i$ in hour $h$ on day-of-week $d$ and month-of-sample $m$, we estimate how indoor $\mathrm{PM}_{2.5}$ varies with contemporaneous and previous hour measurements of outdoor $\mathrm{PM}_{2.5}$ :

$$
P M_{i h d m}^{i n}=\sum_{k=0}^{6} \beta_{k} P M_{i ; h-k ; d m}^{\text {out }}+\gamma_{i}+\delta_{h}+\eta_{d}+\theta_{m}+\varepsilon_{i h d m}
$$

To isolate the contribution of outdoor $\mathrm{PM}_{2.5}$ to indoor $\mathrm{PM}_{2.5}$ from other time varying $\mathrm{PM}_{2.5}$ sources (most notably, indoor-sourced $\mathrm{PM}_{2.5}$ ), we use fixed effects to flexibly control for monthly trends in $\mathrm{PM}_{2.5}$ over the sample $\left(\theta_{m}\right)$ and household-specific average variation in $\mathrm{PM}_{2.5}$ within the day $\left(\delta_{h}\right)$. Day-of-week fixed effects $\left(\eta_{d}\right)$ control for differences in patterns between weekdays and weekends.

We include six lags here (outdoor $\mathrm{PM}_{2.5}$ at each of the previous 6 hours) to account for lingering effects of outdoor concentrations in previous hours on contemporaneous indoor concentrations, although results are robust to the inclusion of additional lags. From this regression we derive an estimate for outdoor-indoor infiltration by calculating the cumulative effect of $1 \mu \mathrm{g} / \mathrm{m}^{3}$ increase in outdoor concentrations on indoor concentrations:

$$
\text { infiltration }=\sum_{k=0}^{6} \beta_{k}
$$

In order to assess the importance of modeling structure we re-estimated Eq. 4 with four different lag structures: a distributed lag model with lags for outdoor $\mathrm{PM}_{2.5}$ only (shown above), a lagged dependent variable model with a lag for indoor $\mathrm{PM}_{2.5}$ only, a model with both indoor and outdoor $\mathrm{PM}_{2.5}$ lags, and finally a model with no lag terms (Table S2). Infiltration rate estimates derived from each of the models are highly similar (Fig S7) and models with more than six lags produce indistinguishable estimates of infiltration rates. 
In order to examine heterogeneity in infiltration rates across hourly outdoor pollution levels and by smoke presence, we first estimate a non-linear version of Equation 4. Namely, we model indoor $\mathrm{PM}_{2.5}$ as a 4th degree polynomial of outdoor $\mathrm{PM}_{2.5}$ (and its lags) and interact it with a dummy variable indicating whether smoke was present. The smoke dummy is defined as 1 when a NOAA HMS plume reported a heavy plume over the PurpleAir monitor on that day and 0 otherwise:

$$
\begin{aligned}
P M_{i h d t}^{\text {in }}= & {\left[\sum_{k=0}^{6} \beta_{k} P M_{i ; h-k ; d m}^{\text {out }}+\sum_{k=0}^{6} \alpha_{k}\left(P M_{i ; h-k ; d m}^{\text {out }}\right)^{2}+\right.} \\
& \left.\sum_{k=0}^{6} v_{k}\left(P M_{i ; h-k ; d m}^{\text {out }}\right)^{3}+\sum_{k=0}^{6} \lambda_{k}\left(P M_{i ; h-k ; d m}^{\text {out }}\right)^{4}\right] \cdot \mathbb{1}(\text { smoke }=1)+ \\
& {\left[\sum_{k=0}^{6} \beta_{k} P M_{i ; h-k ; d m}^{\text {out }}+\sum_{k=0}^{6} \alpha_{k}\left(P M_{i ; h-k ; d m}^{\text {out }}\right)^{2}+\right.} \\
& \left.\sum_{k=0}^{6} v_{k}\left(P M_{i ; h-k ; d m}^{\text {out }}\right)^{3}+\sum_{k=0}^{6} \lambda_{k}\left(P M_{i ; h-k ; d m}^{\text {out }}\right)^{4}\right] \cdot \mathbb{1}(\text { smoke }=0)+ \\
& \gamma_{i}+\delta_{h}+\eta_{d}+\theta_{m}+\varepsilon_{i h d m}
\end{aligned}
$$

To measure the infiltration rate we then calculate the derivative of indoor $\mathrm{PM}_{2.5}$ with respect to outdoor $\mathrm{PM}_{2.5}$ estimated in Equation 6 and evaluate across the 1st-99th percentile of observed hourly outdoor $\mathrm{PM}_{2.5}$ concentrations as well as the indicator for whether or not smoke was present. Responses are plotted in Figure 4a.

We also estimate infiltration rates as a function of median census tract income by estimating Equation 4 with an additional income interaction term:

$$
P M_{i h d m}^{i n}=\left[\sum_{k=0}^{6} \beta_{k} P M_{i ; h-k ; d m}^{\text {out }}\right]+\left[\sum_{k=0}^{6} \beta_{k} P M_{i ; h-k ; d m}^{\text {out }}\right] \cdot \text { income }_{i}+\gamma_{i}+\delta_{h}+\eta_{d}+\theta_{m}+\varepsilon_{i h d m}
$$

Median income data come from the American Community Survey. Each indoor monitor was matched to a census tract and median income was pulled for the most recent available year and updated to 2020 dollars.

Finally, for each indoor monitor we estimated a separate distributed lag model analogous to the 
pooled model in Equation 4:

$$
P M_{i h d m}=\sum_{k=0}^{6} \beta_{i k} P M_{j ; h-k ; m}+\delta_{h}+\eta_{m}+\theta_{d}+\varepsilon_{i h d m}
$$

where $\mathrm{PM}_{2.5}$ at indoor monitor $i$ in hour $h$ on day-of-week $d$ and month-of-sample $m$ is modeled as a function of outdoor $\mathrm{PM}_{2.5}$ in that location in the contemporaneous period and for each of the previous 6 hours. Our estimate of the overall infiltration rate for each monitor, which we denote $\beta_{i}$ is then the sum of coefficients over time from the regression for that monitor (i.e., $\beta_{i}=$ $\left.\sum_{k=0}^{6} \beta_{i k}\right)$.

A large number of previous studies [e.g., $(33,35,38,39)$ among many others] have focused on average indoor-to-outdoor concentration ratios (I/O) as a metric of infiltration. If measured over long time periods, this average I/O ratio does represent the net atmospheric barrier provided by a building, but is inclusive of emissions from indoor sources (which may be either trapped or ventilated) (60). Because indoor-sourced emissions can be large, approaches using I/O to understand infiltration have often focused on periods of the day (typically the middle of the night) when indoor sources are likely to be minimal. However, if households alter other infiltration-relevant behaviors at night (e.g., either keeping windows open, or keeping them closed), then period-specific I/O estimates will not be representative of infiltration during the rest of the day. These differences could be correlated with demographics. For instance, households in higher-crime areas might close their windows at night but open them during the day, and wealthy households with air conditioning in low crime areas could do the reverse; in this case, I/O estimates would understate true average infiltration for the first set of houses and overstate for the second.

Our regression-based approach to infiltration measurement is less likely to suffer from this problem. We characterize infiltration as $\partial I / \partial O$, or the change in indoor concentrations when outdoor concentrations change, and use a regression framework to help isolate the contribution of outdoor $\mathrm{PM}_{2.5}$ to indoor $\mathrm{PM}_{2.5}$ from time-varying indoor $\mathrm{PM}_{2.5}$ sources. Indoor emissions sources will not bias $\partial I / \partial O$ estimates so long as variation in these sources (after conditioning out our rich set of within- and across-day fixed effects) is uncorrelated with outdoor sources, which is plausible. As a result, our regression based estimates represent average infiltration rates throughout the whole day, and are less likely to be biased by remaining sources of indoor emissions.

Our analysis suggests this distinction in measurements is especially important over shorter time scales, and during major perturbations in outdoor pollution, like wildfires (Fig S9). A simple takeaway of this study is thus that $\mathrm{I} / \mathrm{O}$ ratios might not adequately capture concentrations when 
populations are trying to stay indoors during a heavy smoke event.

Understanding variation in household infiltration rates Monitor-specific estimates suggest large variation in infiltration across households (Fig 4c), consistent with earlier work (40). However, since monitor-specific infiltration values are themselves estimates from data, observed variation across monitors could reflect either "true" underlying heterogeneity in infiltration, or could simply reflect sampling variation (or some combination of the two).

To distinguish sampling variation from underlying heterogeneity, we estimate a Bayesian hierarchical model $(61,62)$ that models monitor-specific infiltration estimates as being distributed normally about true monitor-specific infiltration values with estimated monitor-specific sampling variance $\hat{\beta}_{i} \sim N\left(\beta_{i}, \hat{s e}_{i}^{2}\right)$, and true monitor-specific infiltration values as drawn from an underlying normal distribution with unknown mean and variance $\beta_{i} \sim N\left(\beta, \sigma^{2}\right)$.

We obtain posterior monitor-specific infiltration values $\beta_{i}$ by assuming uniform prior distributions for $\beta$ and $\sigma$ and applying Bayes' Rule to update our estimates of $\beta, \sigma$, and the $\beta_{i}$ 's. We use 4 chains each with 2,000 total iterations and 1,000 warm-up iterations (resulting in 4,000 postwarmup draws) to fit the model and characterize the posterior distributions of these variables using $\mathrm{R}$ package rstan (63). For this analysis, we limit our eligible sample to the 1,484 indoor monitors associated with infiltration estimates between 0 and 1 across all PurpleAir $\mathrm{PM}_{2.5}$ corrections and model lag structures. Posterior estimates of the $\beta_{i}$ 's are very similar to "raw" regression based estimates from equation 8 , suggesting nearly all the variation is true underlying variation in infiltration, and not due to sampling variation.

To understand underlying heterogeneity in infiltration, we then match each indoor monitor to a range of house- and neighborhood-specific covariates measuring demographics, neighborhood conditions, environmental conditions, and housing characteristics. We download demographic and neighborhood condition covariates at the Census tract level from the 2019 American Community Survey 5-year estimates via the R package tidycensus. We match each monitor to its overlapping or nearest neighboring Census tract.

Among environmental covariates, mean annual heating degree days and cooling degree days over 2000-2020 are obtained via Google Earth Engine (64) from the PRISM Daily Spatial Climate Dataset (AN81d) (65) at tract level and matched with monitors by overlap or nearest neighbor. Each household's 10-year mean outdoor $\mathrm{PM}_{2.5}$ concentration is calculated from gridded annual $\mathrm{PM}_{2.5}$ estimates (66).

We interpolate housing covariates from georeferenced CoreLogic data on household character- 
istics spanning the universe of residential homes in the US (67). Because individuals geolocate their monitors manually on the PurpleAir platform and might not choose to input a location at their precise residence, we match monitors to CoreLogic single-family homes in four ways, taking the inverse distance weighted average of housing characteristics within 100, 250, or $500 \mathrm{~m}$, or by using values at the nearest neighbor within $1 \mathrm{~km}$ (i.e. the residence in CoreLogic closest to the monitor lat/lon). For consistency, the sample utilized in the analysis is limited to the 1,383 indoor monitors that match with a CoreLogic house within $100 \mathrm{~m}$, regardless of interpolation method. Because housing characteristics tend to be highly correlated, we construct a housing index by averaging standardized values of home value, number of stories, number of baths, number of bedrooms, height, and area, and use the index as a predictor.

We then train flexible ML-based models to predict monitor-specific infiltration rates from matched covariates. We divide our sample into a $75 \%$ training dataset and a $25 \%$ held-out test dataset, splitting train and test within 13 disjoint geographic regions covering the contiguous US to ensure a geograhically balanced split. We train random forest and gradient boosted trees models with manually tuned forest and boosting hyperparameters, respectively, and tree parameters tuned using random search with 3 -fold cross-validation repeated 5 times. We conduct tuning and training for each method of matching monitors and CoreLogic houses for robustness.

We report performance statistics $\left(R^{2}\right)$ on held-out test data, and compute the marginal effect of each predictor by evaluating the predicted effect in the test data of moving from the 5 th percentile to the 95th percentile of the predictor, with all other variables fixed at their mean value. We repeat this evaluation for both random forest and gradient boosted trees models, and for all four ways of spatially matching to housing characteristics.

Understanding representativeness of study samples Our novel social media, search trends, and sensor data data are not necessarily derived from representative samples of individuals. Our inferences about behavioral responses to smoke that we draw from these data will thus also not be representative of the population at large if the behavior of those represented in the data in response to smoke exposure differs from the behavior of those not in the data. While the latter is by definition hard to observe, some progress can be made by understanding which individuals and groups are contributing information to these datasets, and by studying other efforts to validate derived behavioral measures against representative data.

Recent surveys of overall internet use suggest that the vast majority ( $>90 \%$ ) of adult Americans use the internet, with limited differences by race and only moderate differences by income (68). While we are unaware of comprehensive demographic data on search activity, previous work has found that search-based measures of issue salience correlate reasonably well with survey-based 
measures (69) and are predictive of a range of economic phenomena (70). In our data, the fact that we see higher income populations more likely to both search for and purchase (in the case of PurpleAir) health protective technologies is additional validation that income-differentiated search behavior predicts real-world behavior.

Regarding our sentiment analysis, roughly a quarter of American adults are estimated to use Twitter, with limited differences in use by race (slightly higher use by non-White populations) but higher use among younger Americans and higher-income Americans (71). Nevertheless, temporal variation in Twitter-derived sentiment measures appears to validate well against measures from independent survey data (25) and to correlate well with survey-derived measures of behavior (72). Finally, previous work on climate stress demonstrates that Twitter-derived sentiment measures responds similarly to such stress as compared with responses measured in representative vital statistics or survey data $(73,74)$.

Our mobility data are derived from smartphones. Recent data suggest that $\sim 85 \%$ of adult Americans own smartphones (75). As with internet usage, smartphone ownership shows limited differences by race but some differences by income, with wealthier populations somewhat more likely to own a smartphone. Thus our mobility measures will be slightly skewed towards wealthier populations. As shown in Fig S1, wealthier populations on average report spending less time at home in representative survey data.

Our PurpleAir sample, among our many datasets, is likely the most non-representative of the overall American populace, with Census tracts that have PurpleAir monitors in them being wealthier, better educated, and slightly more White than tracts without monitors (45) (Fig S6). This finding is again consistent with our broader finding of income-differentiated responses to wildfire smoke. However, it also means that the variation in infiltration we measure might not accurately describe infiltration rates for lower-income and less educated populations, and better measurement of infiltration among these populations is an urgent research priority.

Acknowledgements We thank the Robert Wood Johnson Foundation for funding, SafeGraph for data access, and thank members of the ECHOLab and seminar participants at Cornell, Columbia, MIT, Stanford, UC Berkeley, and UC Santa Barbara for helpful comments.

Data availability Data and code to replicate all results in the paper will be made available on a Github repo at the time of publication. 


\section{References}

[1] Philip J Landrigan, Richard Fuller, Nereus JR Acosta, Olusoji Adeyi, Robert Arnold, Abdoulaye Bibi Baldé, Roberto Bertollini, Stephan Bose-O'Reilly, Jo Ivey Boufford, Patrick N Breysse, et al. The lancet commission on pollution and health. The lancet, 391(10119):462$512,2018$.

[2] Tamma A Carleton and Solomon M Hsiang. Social and economic impacts of climate. Science, 353(6304), 2016.

[3] Anna Aizer, Janet Currie, Peter Simon, and Patrick Vivier. Do low levels of blood lead reduce children's future test scores? American Economic Journal: Applied Economics, 10(1):307-41, 2018.

[4] Tatyana Deryugina, Nolan Miller, David Molitor, and Julian Reif. Geographic and socioeconomic heterogeneity in the benefits of reducing air pollution in the United States. Environmental and energy policy and the economy, 2(1):157-189, 2021.

[5] Marshall Burke, Solomon M Hsiang, and Edward Miguel. Global non-linear effect of temperature on economic production. Nature, 527(7577):235-239, 2015.

[6] Hans Grönqvist, J Peter Nilsson, and Per-Olof Robling. Understanding how low levels of early lead exposure affect children's life trajectories. Journal of Political Economy, 128(9):3376-3433, 2020.

[7] US Department of Health, Human Services, et al. Theory at a glance: A guide for health promotion practice. National Cancer Institute, 2005.

[8] Michael Greenstone and B Kelsey Jack. Envirodevonomics: A research agenda for an emerging field. Journal of Economic Literature, 53(1):5-42, 2015.

[9] John T Abatzoglou and A Park Williams. Impact of anthropogenic climate change on wildfire across western US forests. Proceedings of the National Academy of Sciences, 113(42):11770-11775, 2016.

[10] Marshall Burke, Anne Driscoll, Sam Heft-Neal, Jiani Xue, Jennifer Burney, and Michael Wara. The changing risk and burden of wildfire in the United States. Proceedings of the National Academy of Sciences, 118(2), 2021.

[11] Matthew D Hurteau, Anthony L Westerling, Christine Wiedinmyer, and Benjamin P Bryant. Projected effects of climate and development on California wildfire emissions through 2100. Environmental science \& technology, 48(4):2298-2304, 2014. 
[12] Jia Coco Liu, Loretta J Mickley, Melissa P Sulprizio, Francesca Dominici, Xu Yue, Keita Ebisu, Georgiana Brooke Anderson, Rafi FA Khan, Mercedes A Bravo, and Michelle L Bell. Particulate air pollution from wildfires in the Western US under climate change. Climatic change, 138(3):655-666, 2016.

[13] Michael Goss, Daniel L Swain, John T Abatzoglou, Ali Sarhadi, Crystal A Kolden, A Park Williams, and Noah S Diffenbaugh. Climate change is increasing the likelihood of extreme autumn wildfire conditions across California. Environmental Research Letters, 15(9):094016, 2020.

[14] Colleen E Reid, Michael Brauer, Fay H Johnston, Michael Jerrett, John R Balmes, and Catherine T Elliott. Critical review of health impacts of wildfire smoke exposure. Environmental health perspectives, 124(9):1334-1343, 2016.

[15] Wayne E Cascio. Wildland fire smoke and human health. Science of the total environment, 624:586-595, 2018.

[16] Rongbin Xu, Pei Yu, Michael J Abramson, Fay H Johnston, Jonathan M Samet, Michelle L Bell, Andy Haines, Kristie L Ebi, Shanshan Li, and Yuming Guo. Wildfires, global climate change, and human health. New England Journal of Medicine, 383(22):2173-2181, 2020.

[17] Xiaodan Zhou, Kevin Josey, Leila Kamareddine, Miah C Caine, Tianjia Liu, Loretta J Mickley, Matthew Cooper, and Francesca Dominici. Excess of COVID-19 cases and deaths due to fine particulate matter exposure during the 2020 wildfires in the United States. Science Advances, 7(33):eabi8789, 2021.

[18] Sam Heft-Neal, Anne Driscoll, Wei Yang, Gary Shaw, and Marshall Burke. Associations between wildfire smoke exposure during pregnancy and risk of preterm birth in California. Environmental Research, 203:111872, 2021.

[19] Francisca N Santana, David JX Gonzalez, and Gabrielle Wong-Parodi. Psychological factors and social processes influencing wildfire smoke protective behavior: insights from a case study in Northern California. Climate Risk Management, page 100351, 2021.

[20] AG Rappold, MC Hano, S Prince, L Wei, SM Huang, C Baghdikian, B Stearns, X Gao, $\mathrm{S}$ Hoshiko, WE Cascio, et al. Smoke sense initiative leverages citizen science to address the growing wildfire-related public health problem. GeoHealth, 3(12):443-457, 2019.

[21] Colleen E Reid, Michael Jerrett, Ira B Tager, Maya L Petersen, Jennifer K Mann, and John R Balmes. Differential respiratory health effects from the 2008 northern California wildfires: a spatiotemporal approach. Environmental research, 150:227-235, 2016. 
[22] Michelle C Kondo, Anneclaire J De Roos, Lauren S White, Warren E Heilman, Miranda H Mockrin, Carol Ann Gross-Davis, and Igor Burstyn. Meta-analysis of heterogeneity in the effects of wildfire smoke exposure on respiratory health in North America. International journal of environmental research and public health, 16(6):960, 2019.

[23] Jeff Wen and Marshall Burke. Wildfire smoke exposure worsens learning outcomes. working paper, 2021.

[24] EPA. Wildfire smoke a guide for public health officials, 2019 revision. US Environmental Protection Agency, 2019.

[25] Max Pellert, Hannah Metzler, Michael Matzenberger, and David Garcia. Validating daily social media macroscopes of emotions. arXiv preprint arXiv:2108.07646, 2021.

[26] Patrick Baylis. Temperature and temperament: Evidence from Twitter. Journal of Public Economics, 184:104161, 2020.

[27] Patrick Baylis, Nick Obradovich, Yury Kryvasheyeu, Haohui Chen, Lorenzo Coviello, Esteban Moro, Manuel Cebrian, and James H Fowler. Weather impacts expressed sentiment. PloS one, 13(4):e0195750, 2018.

[28] Clayton Hutto and Eric Gilbert. Vader: A parsimonious rule-based model for sentiment analysis of social media text. In Proceedings of the International AAAI Conference on Web and Social Media, volume 8, 2014.

[29] Zheye Wang, Xinyue Ye, and Ming-Hsiang Tsou. Spatial, temporal, and content analysis of Twitter for wildfire hazards. Natural Hazards, 83(1):523-540, 2016.

[30] Sonya Sachdeva, Sarah McCaffrey, and Dexter Locke. Social media approaches to modeling wildfire smoke dispersion: spatiotemporal and social scientific investigations. Information, Communication \& Society, 20(8):1146-1161, 2017.

[31] Hyunyoung Choi and Hal Varian. Predicting the present with Google Trends. 88:2-9. _eprint: https://onlinelibrary.wiley.com/doi/pdf/10.1111/j.1475-4932.2012.00809.x.

[32] Sharad Goel, Jake M. Hofman, Sébastien Lahaie, David M. Pennock, and Duncan J. Watts. Predicting consumer behavior with web search. 107(41):17486-17490. Publisher: National Academy of Sciences Section: Physical Sciences. 
[33] Yutong Liang, Deep Sengupta, Mark Joseph Campmier, Joshua Apte, David M Lunderberg, and Allen Goldstein. Wildfire smoke impacts on indoor air quality assessed using crowdsourced data in California. Proceedings of the National Academy of Sciences, 118(36):e2106478118, 2021.

[34] Kristin A Miller, Elizabeth W Spalt, Amanda J Gassett, Cynthia L Curl, Timothy V Larson, Ed Avol, Ryan W Allen, Sverre Vedal, Adam A Szpiro, and Joel D Kaufman. Estimating ambient-origin PM2.5 exposure for epidemiology: observations, prediction, and validation using personal sampling in the Multi-Ethnic Study of Atherosclerosis. Journal of exposure science \& environmental epidemiology, 29(2):227-237, 2019.

[35] Prateek M. Shrestha, Jamie L. Humphrey, Elizabeth J. Carlton, John L. Adgate, Kelsey E. Barton, Elisabeth D. Root, and Shelly L. Miller. Impact of outdoor air pollution on indoor air quality in low-income homes during wildfire seasons. International Journal of Environmental Research and Public Health, 16(19), 2019.

[36] CK Uejio, JD Tamerius, J Vredenburg, G Asaeda, DA Isaacs, J Braun, A Quinn, and JP Freese. Summer indoor heat exposure and respiratory and cardiovascular distress calls in New York City, NY, US. Indoor air, 26(4):594-604, 2016.

[37] Lauren Ferguson, Jonathon Taylor, Michael Davies, Clive Shrubsole, Phil Symonds, and Sani Dimitroulopoulou. Exposure to indoor air pollution across socio-economic groups in high-income countries: A scoping review of the literature and a modelling methodology. Environment international, 143:105748, 2020.

[38] Jianzhao Bi, Lance A Wallace, Jeremy A Sarnat, and Yang Liu. Characterizing outdoor infiltration and indoor contribution of PM2.5 with citizen-based low-cost monitoring data. Environmental Pollution, 276:116763, 2021.

[39] Ryan W Allen, Sara D Adar, Ed Avol, Martin Cohen, Cynthia L Curl, Timothy Larson, LJ Sally Liu, Lianne Sheppard, and Joel D Kaufman. Modeling the residential infiltration of outdoor PM2.5 in the Multi-Ethnic Study of Atherosclerosis and Air Pollution (MESA Air). Environmental health perspectives, 120(6):824-830, 2012.

[40] Benjamin Krebs, Jennifer Burney, Joshua Graff Zivin, and Matthew Neidell. Using crowdsourced data to assess the temporal and spatial relationship between indoor and outdoor particulate matter. Environmental Science \& Technology, 55(9):6107-6115, 2021.

[41] Nolan Miller, David Molitor, and Eric Zou. Blowing smoke: Health impacts of wildfire plume dynamics. working paper, 2017. 
[42] Ana G Rappold, Wayne E Cascio, Vasu J Kilaru, Susan L Stone, Lucas M Neas, Robert B Devlin, and David Diaz-Sanchez. Cardio-respiratory outcomes associated with exposure to wildfire smoke are modified by measures of community health. Environmental Health, 11(1):1-9, 2012.

[43] Robert J Brulle and David N Pellow. Environmental justice: human health and environmental inequalities. Annu. Rev. Public Health, 27:103-124, 2006.

[44] Anjum Hajat, Ana V Diez-Roux, Sara D Adar, Amy H Auchincloss, Gina S Lovasi, Marie S O’Neill, Lianne Sheppard, and Joel D Kaufman. Air pollution and individual and neighborhood socioeconomic status: evidence from the Multi-Ethnic Study of Atherosclerosis (MESA). Environmental health perspectives, 121(11-12):1325-1333, 2013.

[45] Priyanka deSouza and Patrick L Kinney. On the distribution of low-cost PM2.5 sensors in the US: demographic and air quality associations. Journal of Exposure Science \& Environmental Epidemiology, 31(3):514-524, 2021.

[46] Cong Sun, Matthew E Kahn, and Siqi Zheng. Self-protection investment exacerbates air pollution exposure inequality in urban China. Ecological economics, 131:468-474, 2017.

[47] Janet Currie, John Voorheis, and Reed Walker. What caused racial disparities in particulate exposure to fall? new evidence from the Clean Air Act and satellite-based measures of air quality. Technical report, National Bureau of Economic Research, 2020.

[48] Qian Di, Heresh Amini, Liuhua Shi, Itai Kloog, Rachel Silvern, James Kelly, M Benjamin Sabath, Christine Choirat, Petros Koutrakis, Alexei Lyapustin, et al. An ensemble-based model of PM2.5 concentration across the contiguous United States with high spatiotemporal resolution. Environment international, 130:104909, 2019.

[49] Colleen E Reid, Ellen M Considine, Melissa M Maestas, and Gina Li. Daily PM2.5 concentration estimates by county, zip code, and census tract in 11 western states 2008-2018. Scientific data, 8(1):1-15, 2021.

[50] Katelyn O’Dell, Bonne Ford, Emily V Fischer, and Jeffrey R Pierce. Contribution of wildland-fire smoke to US PM2.5 and its influence on recent trends. Environmental science \& technology, 53(4):1797-1804, 2019.

[51] US Census Bureau. "Characteristics of people by language spoken at home 2019" 20152019 american community survey 5-year estimates. accessed via $\mathrm{R}$ package tidycensus. 
[52] Ryan Fox Squire. Measuring and correcting sampling bias in SafeGraph patterns for more accurate demographic analysis.

[53] Ryan Fox Squire. Quantifying sampling bias in SafeGraph patterns.

[54] Ryan Fox Squire. What about bias in the SafeGraph dataset?

[55] Joakim A Weill, Matthieu Stigler, Olivier Deschenes, and Michael R Springborn. Social distancing responses to COVID-19 emergency declarations strongly differentiated by income. Proceedings of the National Academy of Sciences, 117(33):19658-19660, 2020.

[56] Danny Valdez, Marijn Ten Thij, Krishna Bathina, Lauren A Rutter, and Johan Bollen. Social media insights into US mental health during the COVID-19 pandemic: longitudinal analysis of Twitter data. Journal of medical Internet research, 22(12):e21418, 2020.

[57] Lidia Morawska, Julian W Tang, William Bahnfleth, Philomena M Bluyssen, Atze Boerstra, Giorgio Buonanno, Junji Cao, Stephanie Dancer, Andres Floto, Francesco Franchimon, et al. How can airborne transmission of COVID-19 indoors be minimised? Environment international, 142:105832, 2020.

[58] Karoline K Barkjohn, Brett Gantt, and Andrea L Clements. Development and application of a United States-wide correction for PM2.5 data collected with the PurpleAir sensor. Atmospheric Measurement Techniques, 14(6):4617-4637, 2021.

[59] Lance Wallace, Jianzhao Bi, Wayne R Ott, Jeremy Sarnat, and Yang Liu. Calibration of low-cost PurpleAir outdoor monitors using an improved method of calculating PM2.5. Atmospheric Environment, 256:118432, 2021.

[60] Chun Chen and Bin Zhao. Review of relationship between indoor and outdoor particles: I/O ratio, infiltration factor and penetration factor. Atmospheric environment, 45(2):275-288, 2011.

[61] Rachael Meager. Understanding the average impact of microcredit expansions: A bayesian hierarchical analysis of seven randomized experiments. American Economic Journal: Applied Economics, 11(1):57-91, January 2019.

[62] Eva Vivalt. How much can we generalize from impact evaluations? Journal of the European Economic Association, 18(6):3045-3089, 2020.

[63] Stan Development Team. RStan: the R interface to Stan, 2020. R package version 2.21.2. 
[64] Noel Gorelick, Matt Hancher, Mike Dixon, Simon Ilyushchenko, David Thau, and Rebecca Moore. Google Earth Engine: Planetary-scale geospatial analysis for everyone. Remote Sensing of Environment, 2017.

[65] PRISM Climate Group, Oregon State University. Prism daily spatial climate dataset an81d. Accessed via Google Earth Engine on 28 April 2021.

[66] Aaron Van Donkelaar, Randall V Martin, Chi Li, and Richard T Burnett. Regional estimates of chemical composition of fine particulate matter using a combined geoscience-statistical method with information from satellites, models, and monitors. Environmental science \& technology, 53(5):2595-2611, 2019.

[67] CoreLogic. Corelogic tax and deed data. Accessed via Stanford Libraries.

[68] Pew Research Center. Internet/broadband fact sheet. https://www.pewresearch.org/internet/fact-sheet/internet-broadband/, 2021.

[69] Jonathan Mellon. Internet search data and issue salience: The properties of Google Trends as a measure of issue salience. Journal of Elections, Public Opinion \& Parties, 24(1):45$72,2014$.

[70] Hyunyoung Choi and Hal Varian. Predicting the present with Google Trends. Economic record, 88:2-9, 2012.

[71] Brooke Auxier and Monica Anderson. Social media use in 2021. Pew Research Center, 2021.

[72] Jared Jashinsky, Scott H Burton, Carl L Hanson, Josh West, Christophe Giraud-Carrier, Michael D Barnes, and Trenton Argyle. Tracking suicide risk factors through Twitter in the US. Crisis, 2014.

[73] Marshall Burke, Felipe González, Patrick Baylis, Sam Heft-Neal, Ceren Baysan, Sanjay Basu, and Solomon Hsiang. Higher temperatures increase suicide rates in the United States and Mexico. Nature climate change, 8(8):723-729, 2018.

[74] Nick Obradovich, Robyn Migliorini, Martin P Paulus, and Iyad Rahwan. Empirical evidence of mental health risks posed by climate change. Proceedings of the National Academy of Sciences, 115(43):10953-10958, 2018.

[75] Pew Research Center. Mobile fact sheet. https://www.pewresearch.org/internet/factsheet/mobile/, 2021. 


\section{Supplemental information}
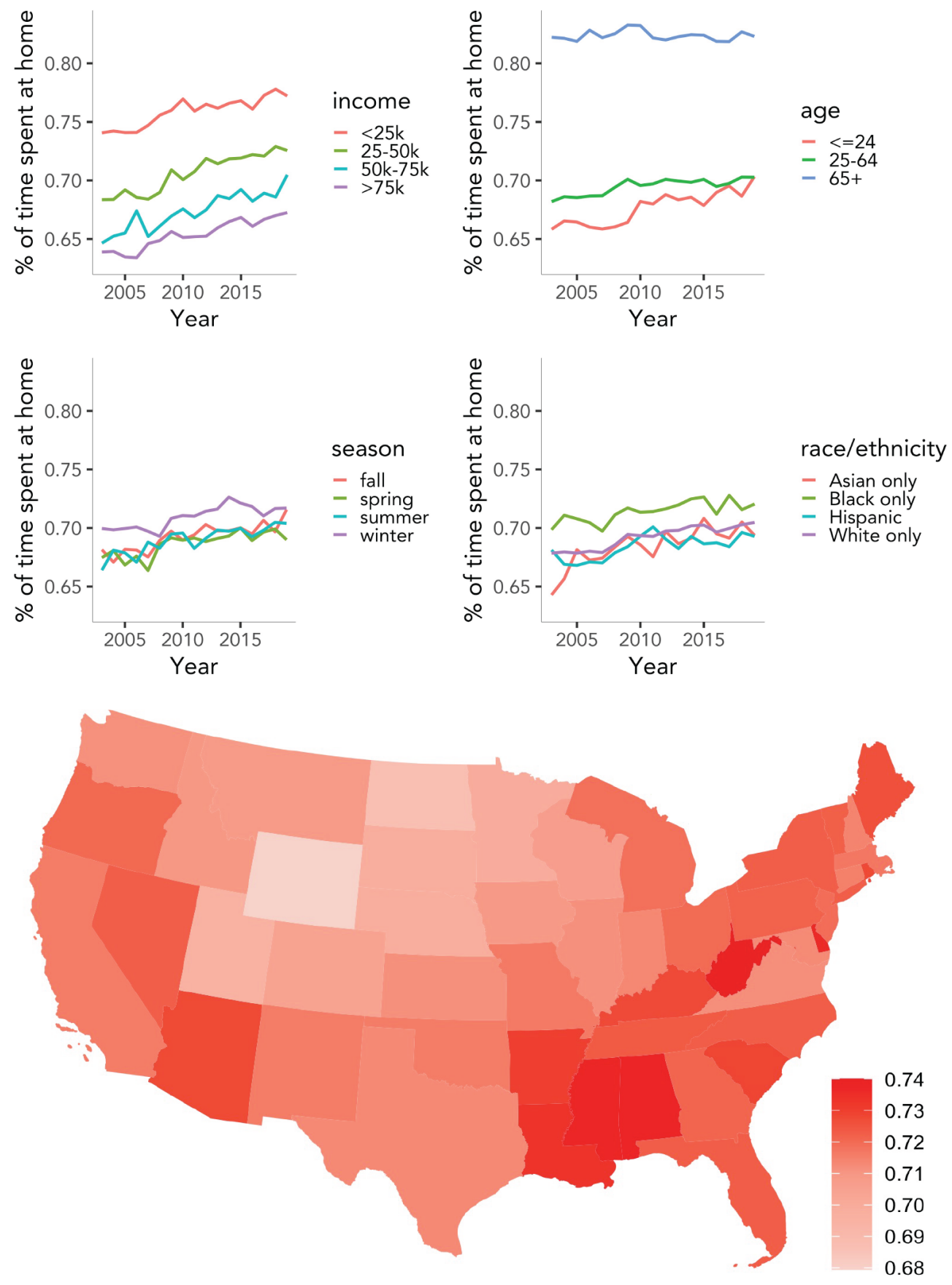

Figure S1: Time spent indoors at home in America. Data are from repeated rounds of the American Time Use Survey. Top panels show data by average income, age, season and race/ethnicity. Bottom map shows averages by state. 


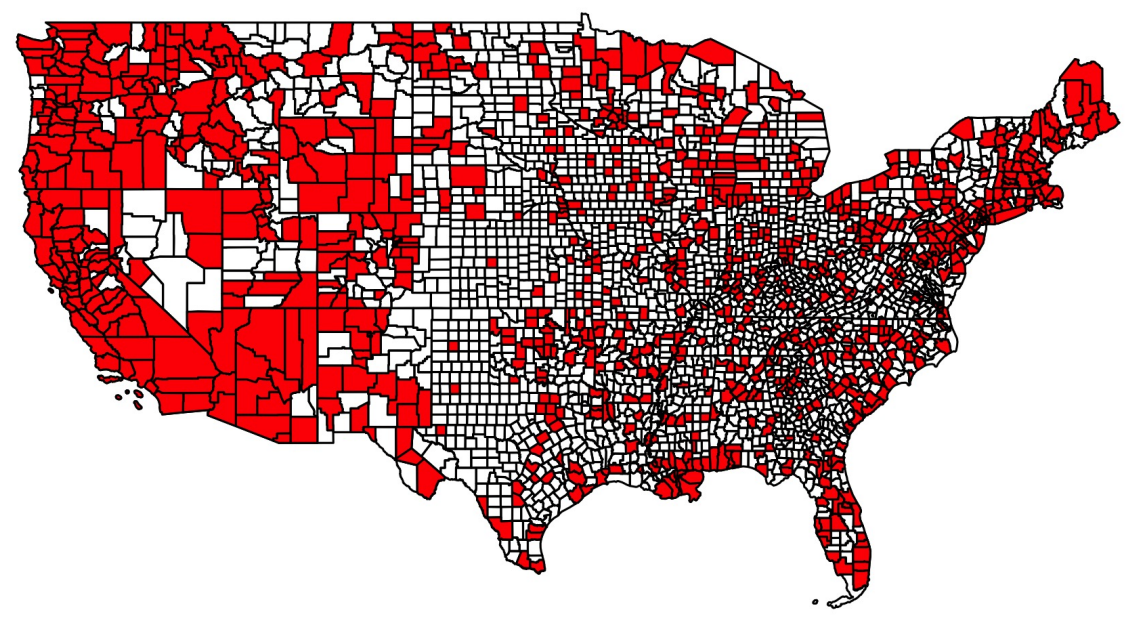

Figure S2: Counties included in analyses that use EPA pollution monitors. Counties in red are those with EPA pollution monitors from which we construct smoke $\mathrm{PM}_{2.5}$ measures for the behavioral analyses. 


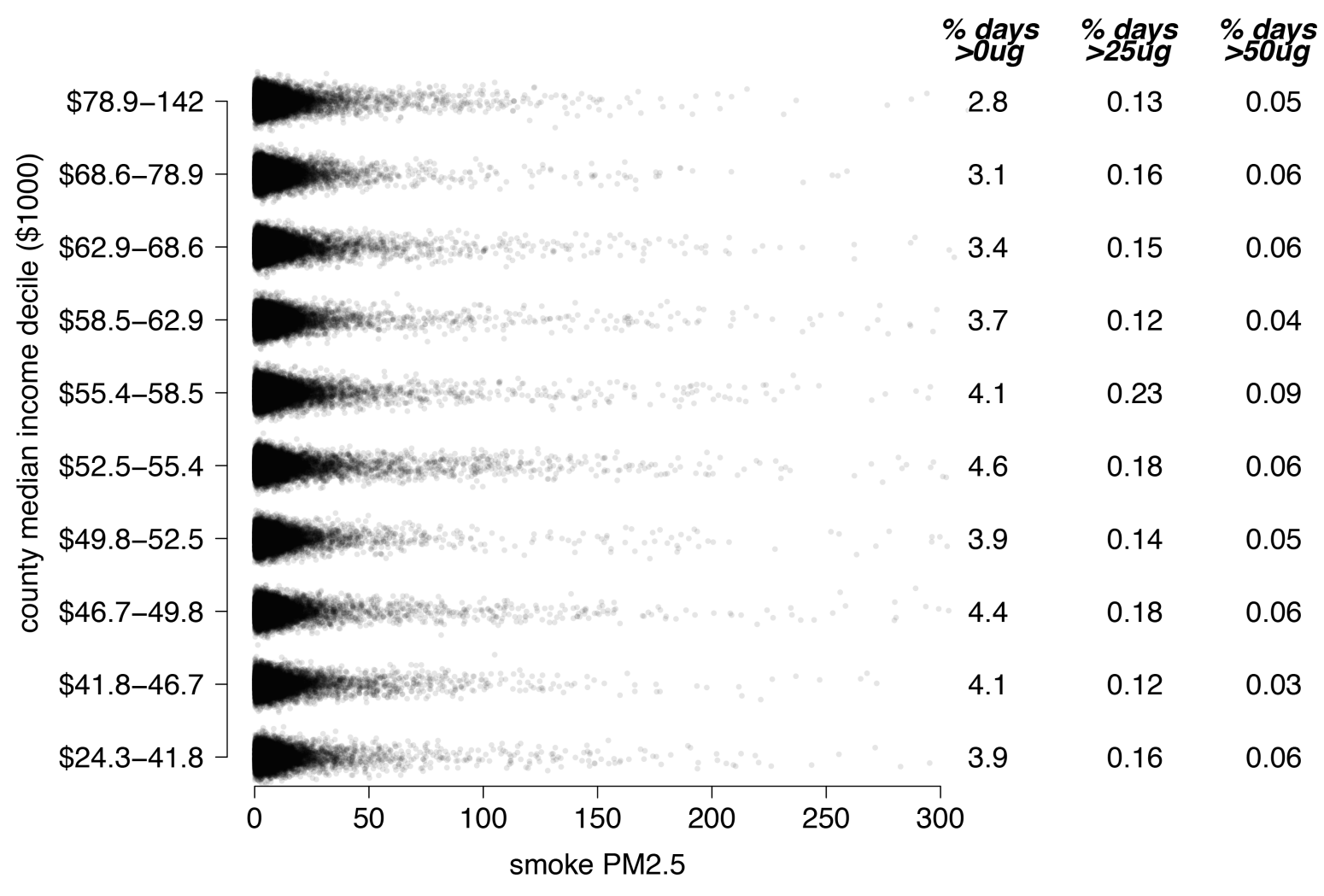

Figure S3: Exposure to average and acute smoke $\mathbf{P M}_{2.5}$ at the county level does not differ systematically by income. Daily smoke $\mathrm{PM}_{2.5}$ exposures by income decile across US counties, 2006-2020. Dots represent daily observations where smoke $\mathrm{PM}_{2.5}$ was non-zero. Plot is truncated at $300 \mathrm{ug}$ for clarity; not plotted are 71 days $\left(0.001 \%\right.$ of the sample) in which smoke $\mathrm{PM}_{2.5}$ exceeded 300. Statistics at right show the percent of observations across the study period with daily smoke $\mathrm{PM}_{2.5}$ observations above the listed value. 

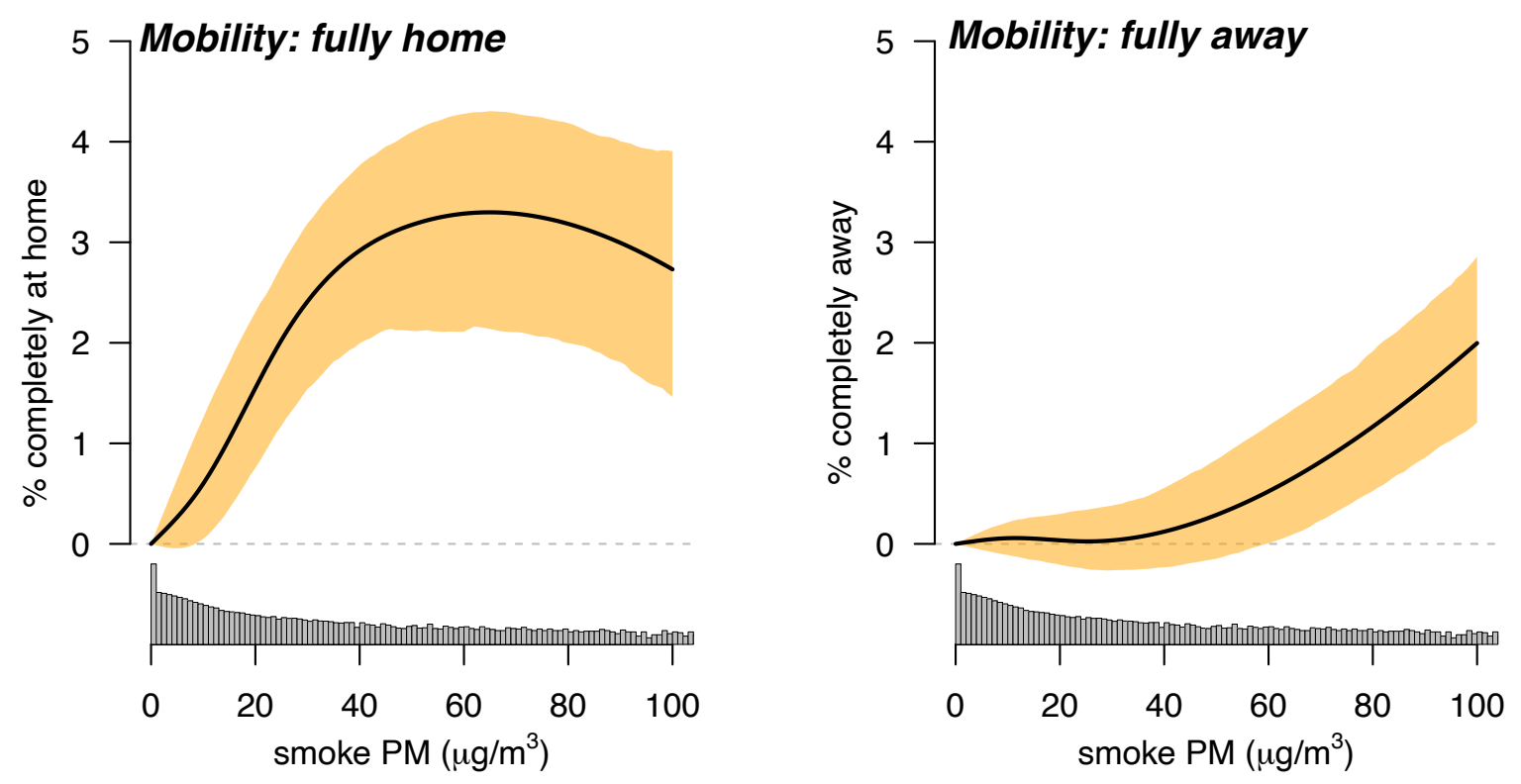

Figure S4: Effect of smoke $\mathbf{P M}_{2.5}$ on percent fully home versus percent fully away. Left plot is as in Fig 2d, right plot is same but for \% fully away from home on that day.
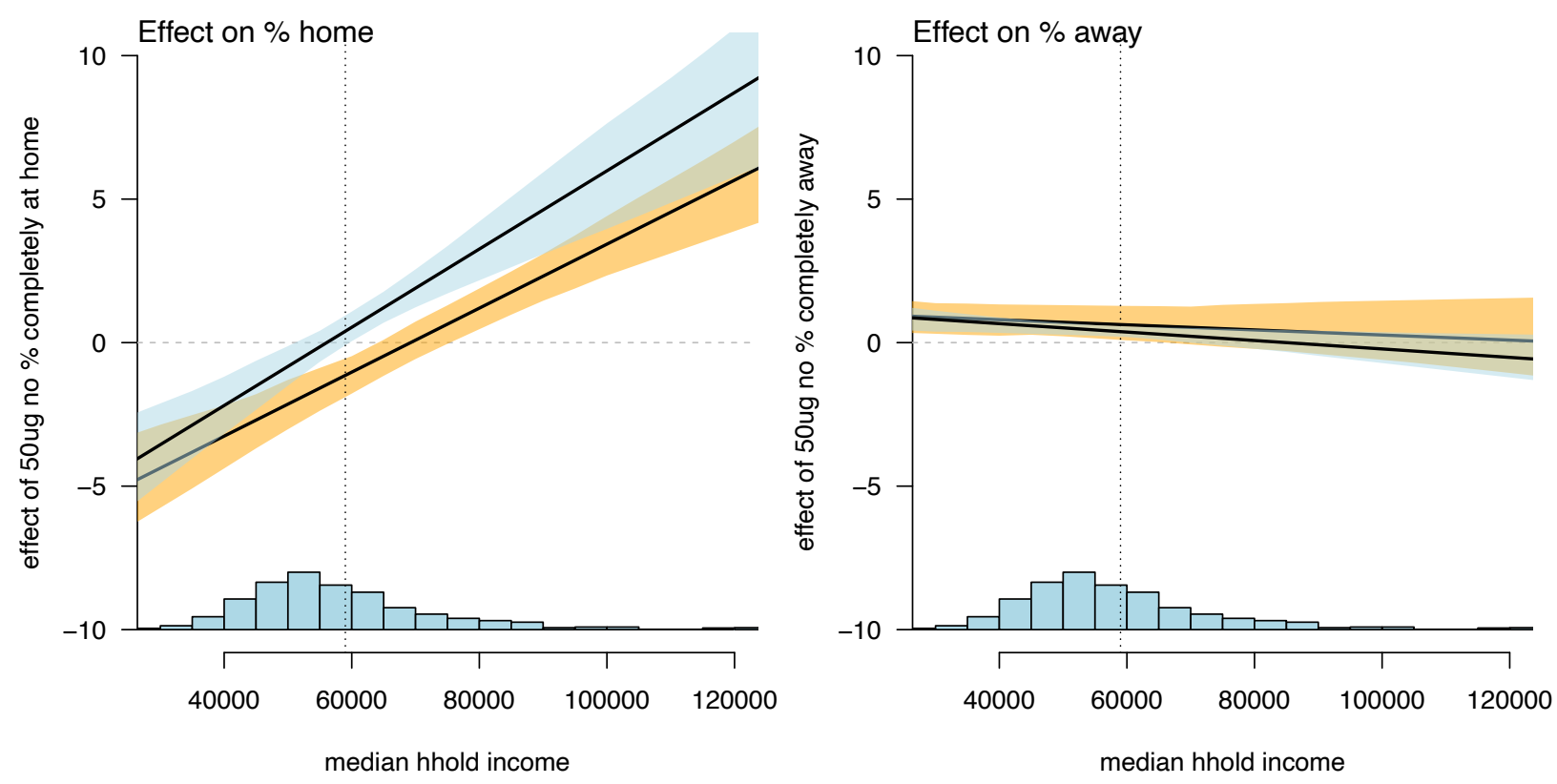

Figure S5: Effect of smoke $\mathbf{P M}_{2.5}$ on mobility as a function of income. Lines show the marginal effect of a heavy smoke exposure (50ug smoke $\mathrm{PM}_{2.5}$ on that day) on percent of individuals completely at home on that day (left plot) or completely away from home (right plot), as a function of median household income in that county. Colors represent models run with either date fixed effects (blue) or state-by-date fixed effects (orange). Shaded area is bootstrapped $95 \%$ CI. 


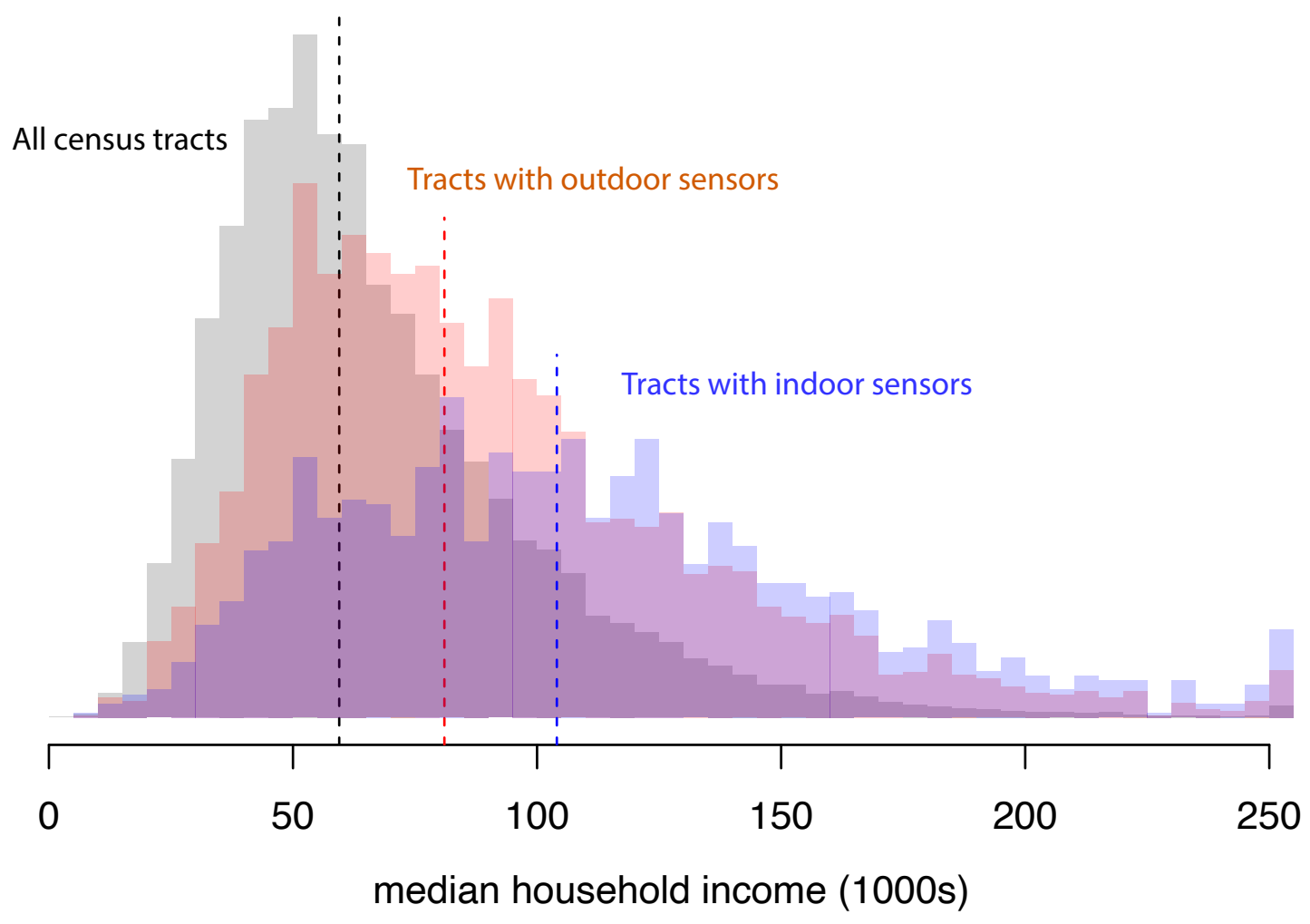

Figure S6: Higher income US census tracts are more likely to have PurpleAir monitors.

Grey bars shows the distribution of tract-level median household income across all US census tracts in the continguous US, red bars the income in tracts with at least one outdoor PurpleAir sensor, and blue bars the income in tracts with at least one indoor PurpleAir sensor. Vertical lines give the median of each distribution. 


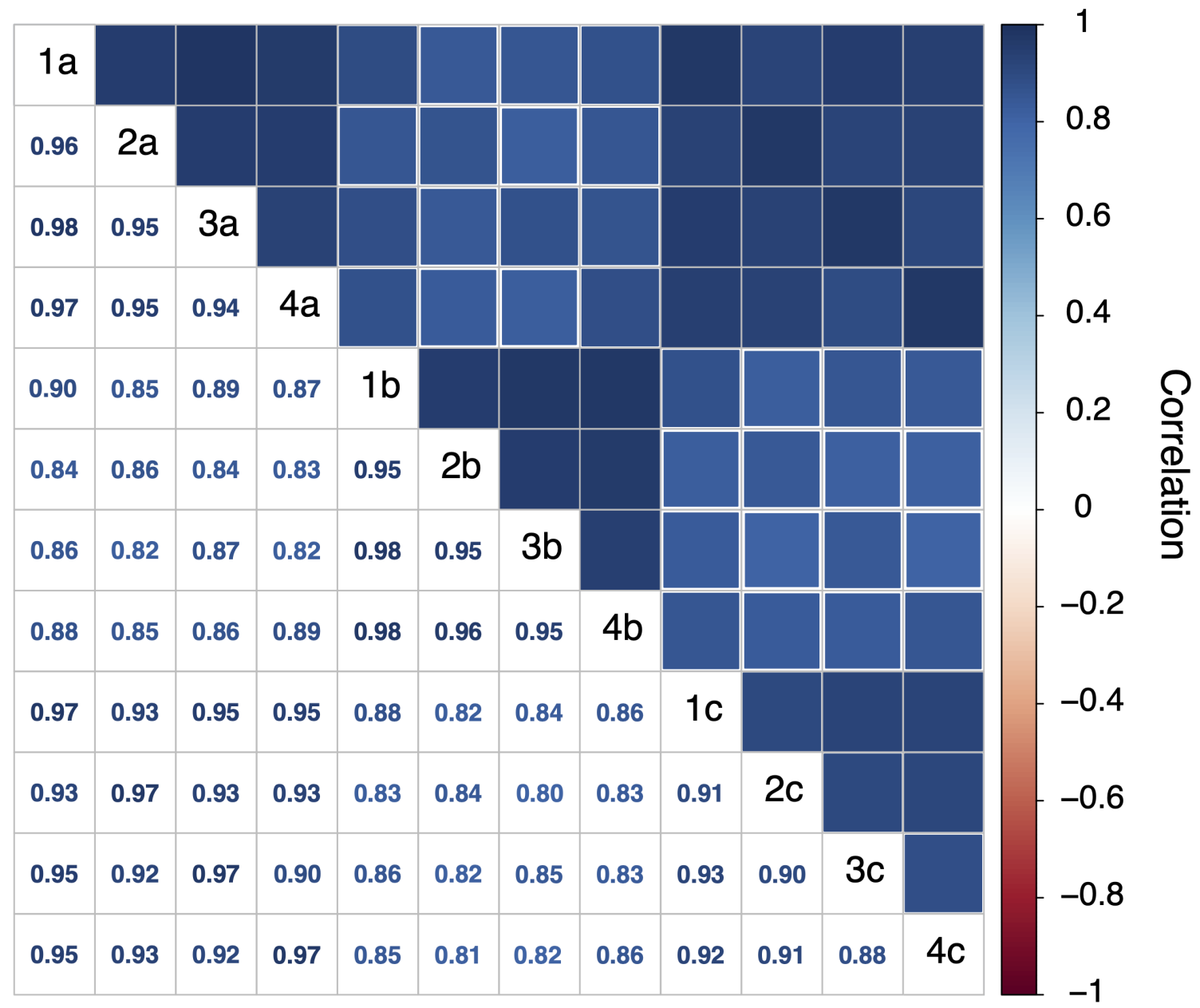

\section{Model}

1. Distributed Lag

2. Lagged Dependent Variable

3. No Lags

4. Both Dependent and Independent Variable Lags

\section{$\mathrm{PM}_{2.5}$ Measurements}
a. Derived from particle counts
b. Barkjohn et al 2021 corrected
c. No correction

Figure S7: Infiltration estimates are highly correlated across alternate statistical models and methods of deriving $\mathbf{P M}_{2.5}$ concentrations from Purple Air data. Correlation between infiltration estimates from statistical models with different lag structures (see Table S2 for model equations) and different $\mathrm{PM}_{2.5}$ concentration estimates (see Methods for details). 1a is our preferred specification presented in the main results. 


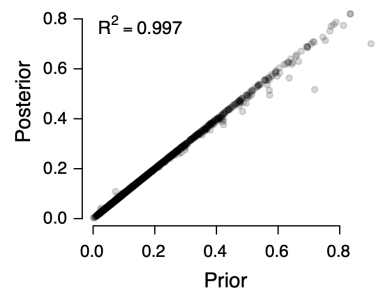

b. Explained variation in infiltration
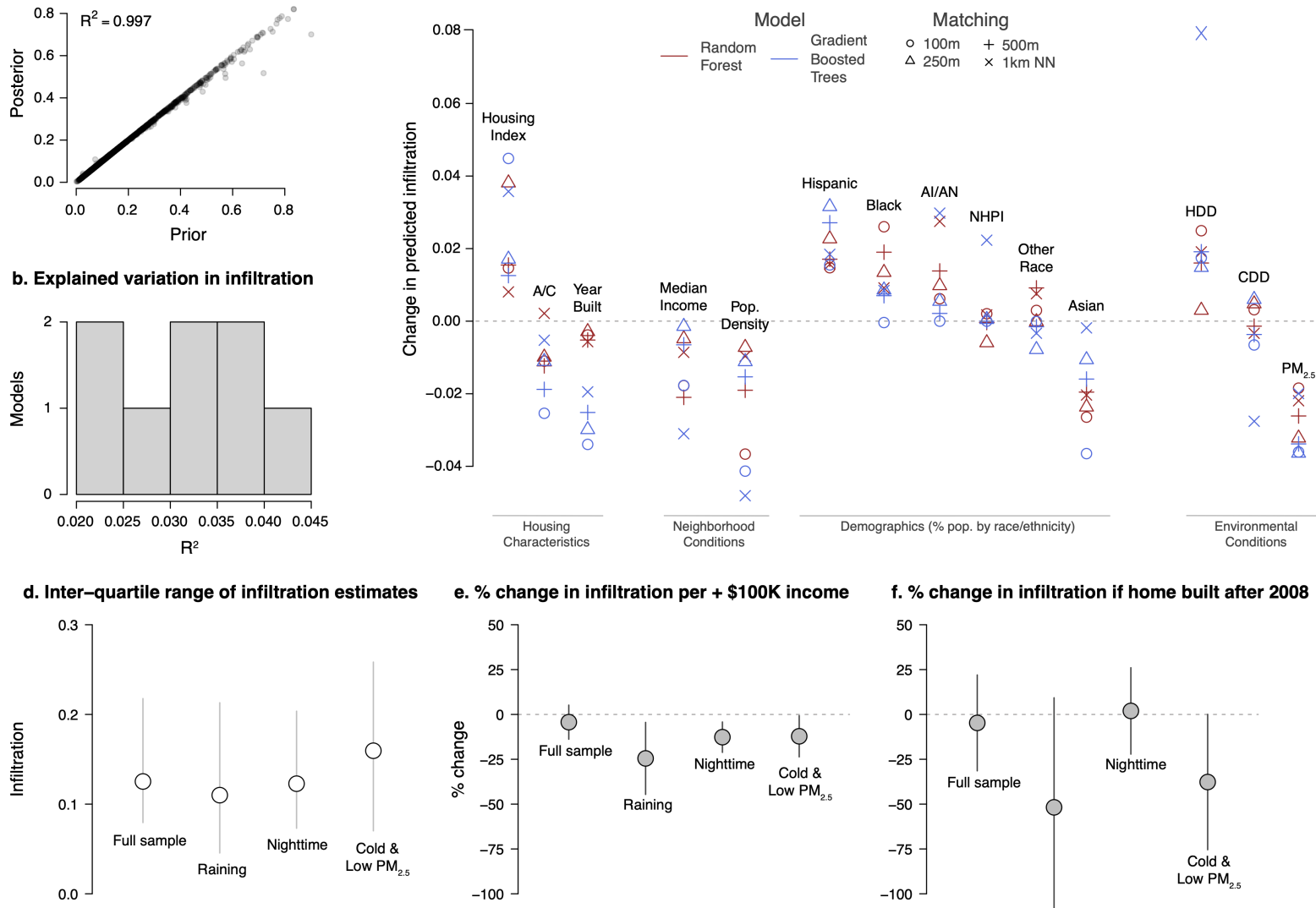

Conditions

f. \% change in infiltration if home built after 2008

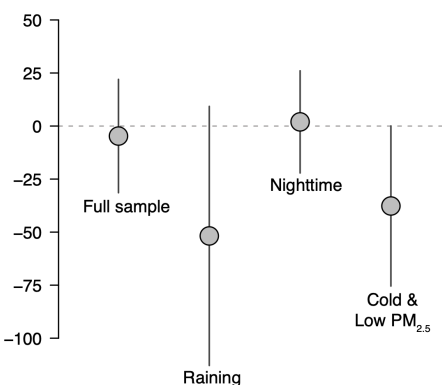

Figure S8: Understanding variation in household-specific infiltration estimates. a. Posterior estimates of monitor-specific infiltration rates from a Bayesian hierarchical model are very similar to "raw" estimates from our monitor-specific time-series regressions, indicating that true heterogeneity rather than sampling noise is what is driving observed differences in estimated infiltration. b. Ability of random forest (RF) or gradient boosted trees (GBT) model to explain variation $\left(R^{2}\right)$ in infiltration across monitors remains low; models use predictors in (c). c. For each predictor, we calculate the effect on infiltration of moving from the 5th to the 95th percentile of that predictor in the test dataset, holding the other predictors constant at their average value in the test dataset; estimates are shown for RF and GBT models and for four alternate spatial buffers used to construct housing predictors. Housing Index is constructed by averaging standardized values of home value, number of stories, number of baths, number of bedrooms, height, and area. A/C measures the inverse distance weighted proportion of matched CoreLogic houses that have air conditioning. Median Income is the median household income in the Census tract population. Race variables (i.e. all demographic covariates except Hispanic) are measured among the nonHispanic/Latino population. AI/AN stands for American Indian and Alaska Native. NHPI stands for Native Hawaiian and other Pacific Islander. HDD and CDD stand for heating degree days and cooling degree days, respectively. d Range of household-level infiltration estimates for the full sample and for sub-samples when behavior (ie opening/closing of doors, use of air purifier) is expected to matter less: when it's raining, nighttime, and periods when it is cold $(<10 \mathrm{C})$ and low$\mathrm{PM}_{2.5}\left(<30 \mu \mathrm{g} / \mathrm{m}^{3}\right)$. e-f during periods when behavioral factors are more likely minimized, infiltration varies more strongly with income and housing age. 


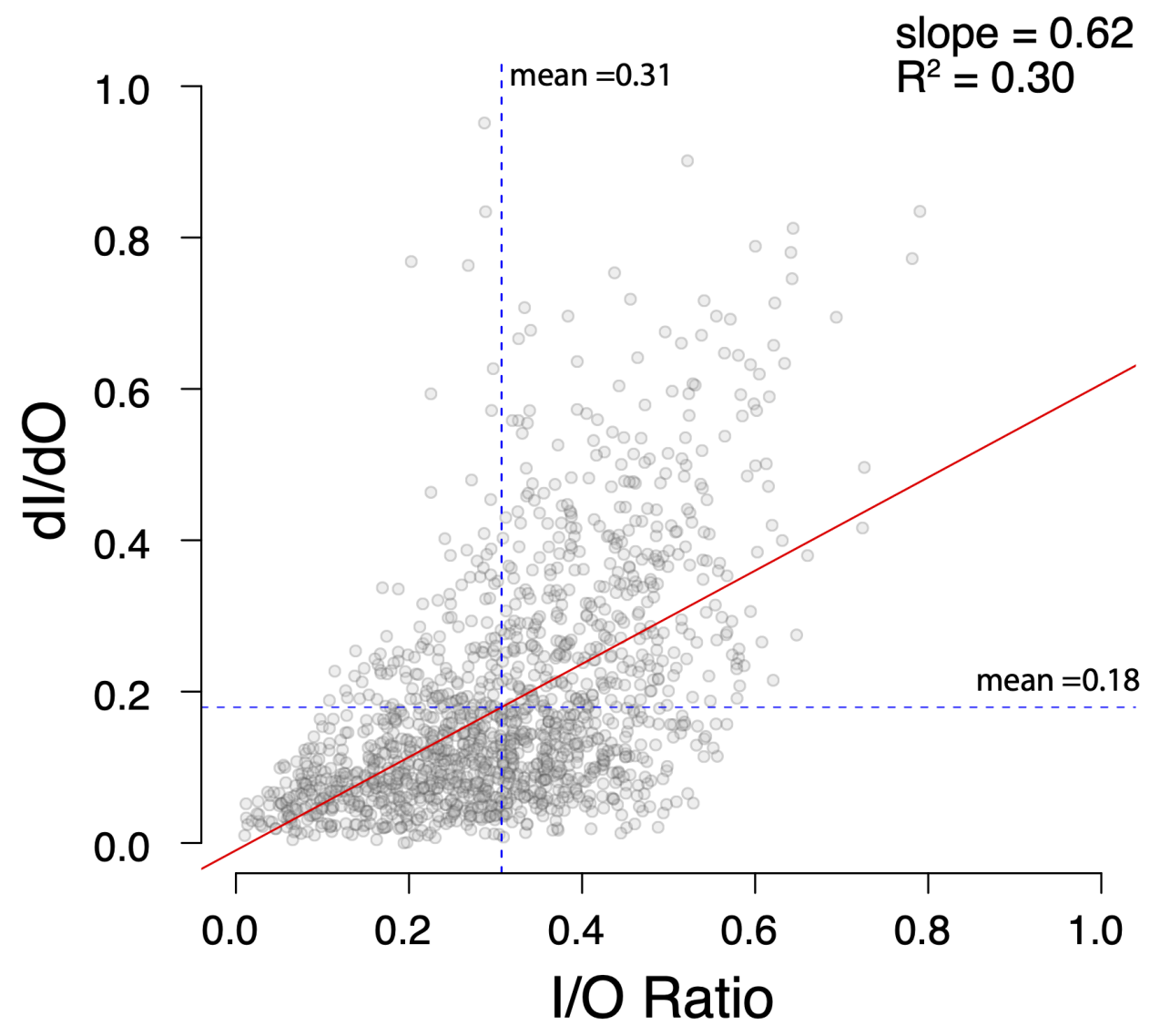

Figure S9: Monitor-specific infiltration estimates using indoor/outdoor ratios versus regression-based approaches. I/O estimates are only modestly correlated with our preferred regression-based estimates that measure the marginal effect on indoor $\mathrm{PM}_{2.5}$ concentrations of a unit increase in outdoor concentrations. For each monitor $\mathrm{I} / \mathrm{O}$ ratio was calculated across all observations with hourly indoor $\mathrm{PM}_{2.5} \leq$ outdoor $\mathrm{PM}_{2.5} . \partial I / \partial O$ was estimated using Eq. 8 as described in Methods. 


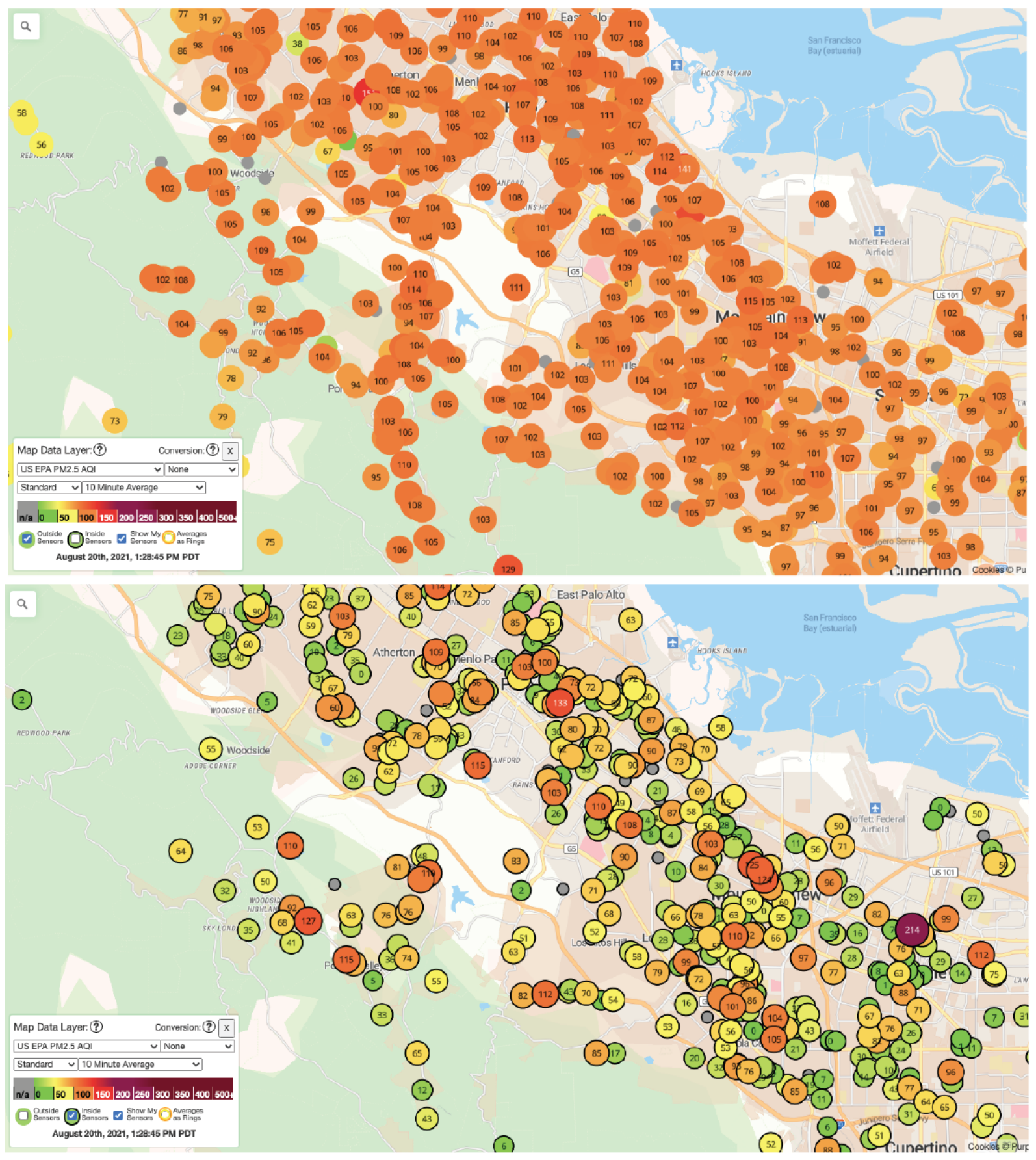

Figure S10: Outdoor and indoor $\mathbf{P M}_{2.5}$ concentrations on a smoke day in CA. Very similar outdoor $\mathrm{PM}_{2.5}$ concentrations during a smoke event on Aug 20th, 2021 over a high-income area of the peninsular Bay Area were associated with widely varying contemporaneous indoor $\mathrm{PM}_{2.5}$ concentrations. 


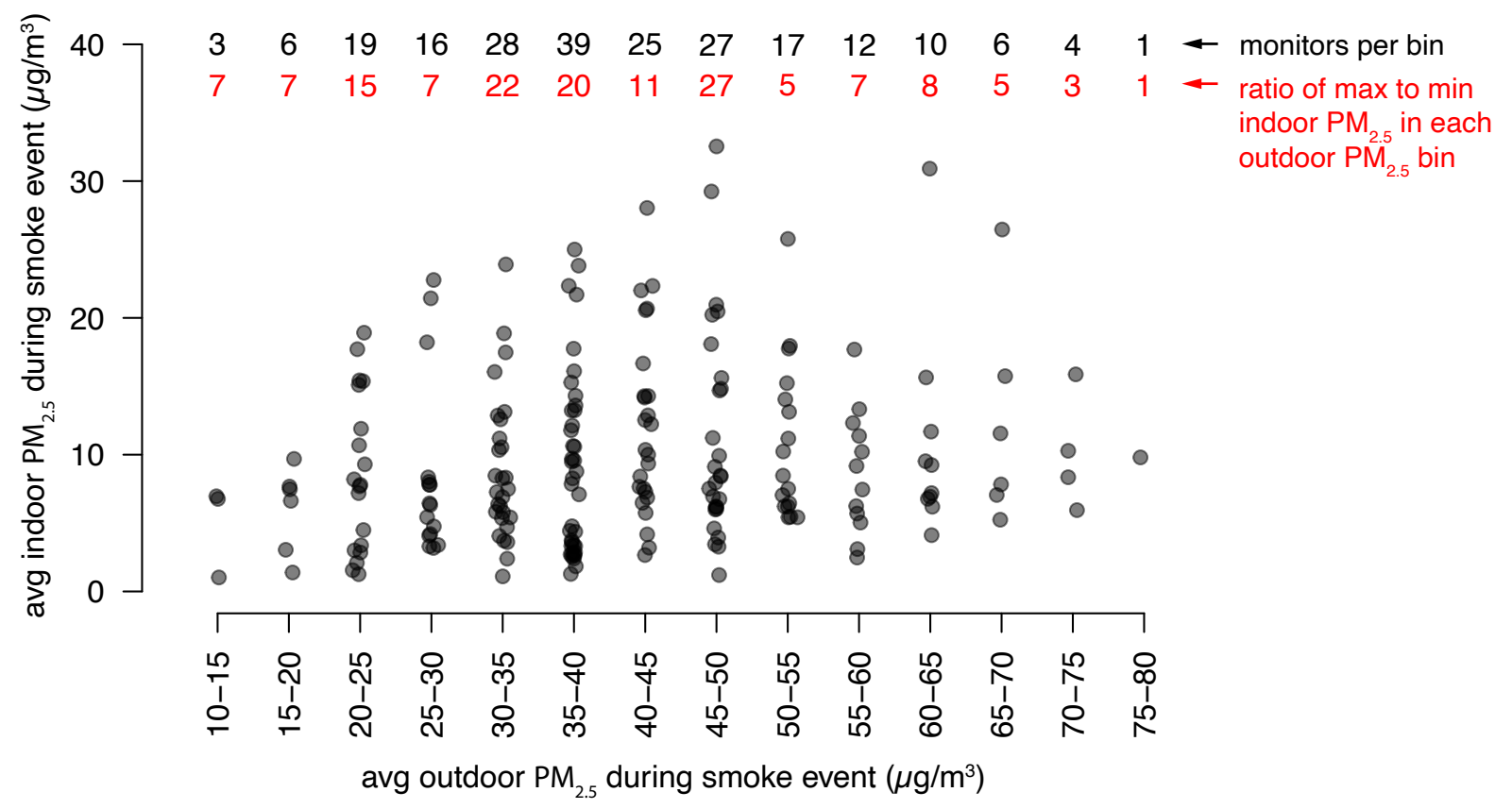

Figure S11: Variation in indoor $\mathbf{P M}_{2.5}$ across monitors with similar outdoor $\mathbf{P M}_{2.5}$ during the Aug/Sep 2020 smoke event in the Bay Area. Each dot is average outdoor $\mathrm{PM}_{2.5}$ and average indoor $\mathrm{PM}_{2.5}$ for an individual monitor in the Bay Area over the Aug/Sep 2020 smoke event, with monitors grouped into $5 \mu \mathrm{g}$ bins based on outdoor exposure. Numbers at top show the number of monitors in each bin (black), and the ratio of maximum to minimum indoor $\mathrm{PM}_{2.5}$ across monitors within each outdoor $\mathrm{PM}_{2.5}$ bin (red). Monitors with average outdoor $\mathrm{PM}_{2.5}$ exposures within $5 \mu \mathrm{g}$ of each other experienced $>20 \mathrm{x}$ differences in indoor $\mathrm{PM}_{2.5}$ exposures. 
Table S1: Hourly EPA reference monitor $\mathbf{P M}_{2.5}$ concentrations versus different constructions of Purple Air $\mathbf{P M}_{2.5}$ concentrations. Each column is a separate linear regression with hourly $\mathrm{PM}_{2.5}$ concentration at an EPA reference monitor as the dependent variable and a different version of Purple Air derived $\mathrm{PM}_{2.5}$ concentration as the independent variable. All models include EPA monitor fixed effects, hour-of-day fixed effects, and month-of-sample fixed effects.

\begin{tabular}{lccc} 
Purple Air PM $_{2.5}$ Construction: & CF_1 $_{1}$ & Barkjohn et al correction & particle count derived \\
\hline Purple Air $\mathrm{PM}_{2.5}$ & $0.600^{* * * *}$ & $1.064^{* * *}$ & $2.196^{* * * *}$ \\
& $(0.035)$ & $(0.069)$ & $(0.109)$ \\
\hline $\mathrm{R}^{2}$ & 0.67 & 0.68 & 0.72 \\
Num.Obs. & 199,394 & 199,394 & 199,394 \\
FE: EPA monitor & $\mathrm{X}$ & $\mathrm{X}$ & $\mathrm{X}$ \\
FE: hour-of-day & $\mathrm{X}$ & $\mathrm{X}$ & $\mathrm{X}$ \\
FE: month-of-sample & $\mathrm{X}$ & $\mathrm{X}$ & $\mathrm{X}$ \\
\hline
\end{tabular}

$* \mathrm{p}<0.05, * * \mathrm{p}<0.01, * * * \mathrm{p}<0.001$ 
Table S2: Models of infiltration

\begin{tabular}{|c|c|c|c|}
\hline & Model description & & Equation \\
\hline 1 & Distributed Lag & $\begin{array}{l}\text { Regression: } \\
\text { Infiltration } \\
\text { measure: }\end{array}$ & $\begin{array}{c}P M_{i h d m}^{\text {in }}=\sum_{k=0}^{6} \alpha_{k} P M_{i, h-k, d m}^{\text {out }}+\gamma_{i}+\delta_{h}+\eta_{d}+\theta_{m}+\varepsilon_{i h d m} \\
\sum_{k=0}^{6} \alpha_{k}\end{array}$ \\
\hline 2 & $\begin{array}{c}\text { Lagged Dependent } \\
\text { Variable }\end{array}$ & $\begin{array}{l}\text { Regression: } \\
\text { Infiltration } \\
\text { measure: }\end{array}$ & $\begin{array}{c}P M_{i h d m}^{\text {in }}=\alpha P M_{i h d m}^{\text {out }}+\beta P M_{i, h-1, d m}^{\text {in }}+\gamma_{i}+\delta_{h}+\eta_{d}+\theta_{m}+\varepsilon_{i h d m} \\
\alpha \cdot\left(\sum_{k=0}^{\infty} \beta^{k}\right)\end{array}$ \\
\hline 3 & No Lags & $\begin{array}{l}\text { Regression: } \\
\text { Infiltration } \\
\text { measure: }\end{array}$ & $\begin{array}{c}P M_{i h d m}^{\text {in }}=\alpha P M_{i h d m}^{\text {out }}+\gamma_{i}+\delta_{h}+\eta_{d}+\theta_{m}+\varepsilon_{i h d m} \\
\alpha\end{array}$ \\
\hline 4 & All Lags & $\begin{array}{l}\text { Regression: } \\
\text { Infiltration } \\
\text { measure: }\end{array}$ & $\begin{aligned} P M_{i h d m}^{i n}= & \sum_{k=0}^{6} \alpha_{k} P M_{i, h-k, d m}^{\text {out }}+\beta P M_{i, h-1, d m}^{i n}+ \\
& \gamma_{i}+\delta_{h}+\eta_{d}+\theta_{m}+\varepsilon_{i h d m} \\
& \left(\sum_{k=0}^{6} \alpha_{k}\right) \cdot\left(\sum_{k=0} \beta^{k}\right)\end{aligned}$ \\
\hline
\end{tabular}


Table S3: Salience results are robust to alternate search words in English and Spanish. Each column is a separate linear regression using the phrase in the column header as the search term, and smoke $\mathrm{PM}_{2.5}$ as the regressor. All models include the same fixed effects but different numbers of observations, as Google Trends does not return information for areas where search volume was low. Observations are marketing area by week. Standard errors are shown in parentheses, clustered at marketing area month.

\begin{tabular}{lcccc}
\multicolumn{1}{c}{ Outcome: } & air quality & smoke & calidad del aire & humo \\
\hline smoke $\mathrm{PM}_{2.5}$ & $0.689^{* * *}$ & $0.567^{* * *}$ & $0.622^{* * *}$ & $0.118^{*}$ \\
& $(0.095)$ & $(0.052)$ & $(0.114)$ & $(0.060)$ \\
\hline Num.Obs. & 50600 & 51900 & 5220 & 22707 \\
FE: date & $\mathrm{X}$ & $\mathrm{X}$ & $\mathrm{X}$ & $\mathrm{X}$ \\
FE: DMA x month & $\mathrm{X}$ & $\mathrm{X}$ & $\mathrm{X}$ & $\mathrm{X}$ \\
\hline
\end{tabular}

$+\mathrm{p}<0.1,{ }^{*} \mathrm{p}<0.05, * * \mathrm{p}<0.01, * * * \mathrm{p}<0.001$ 


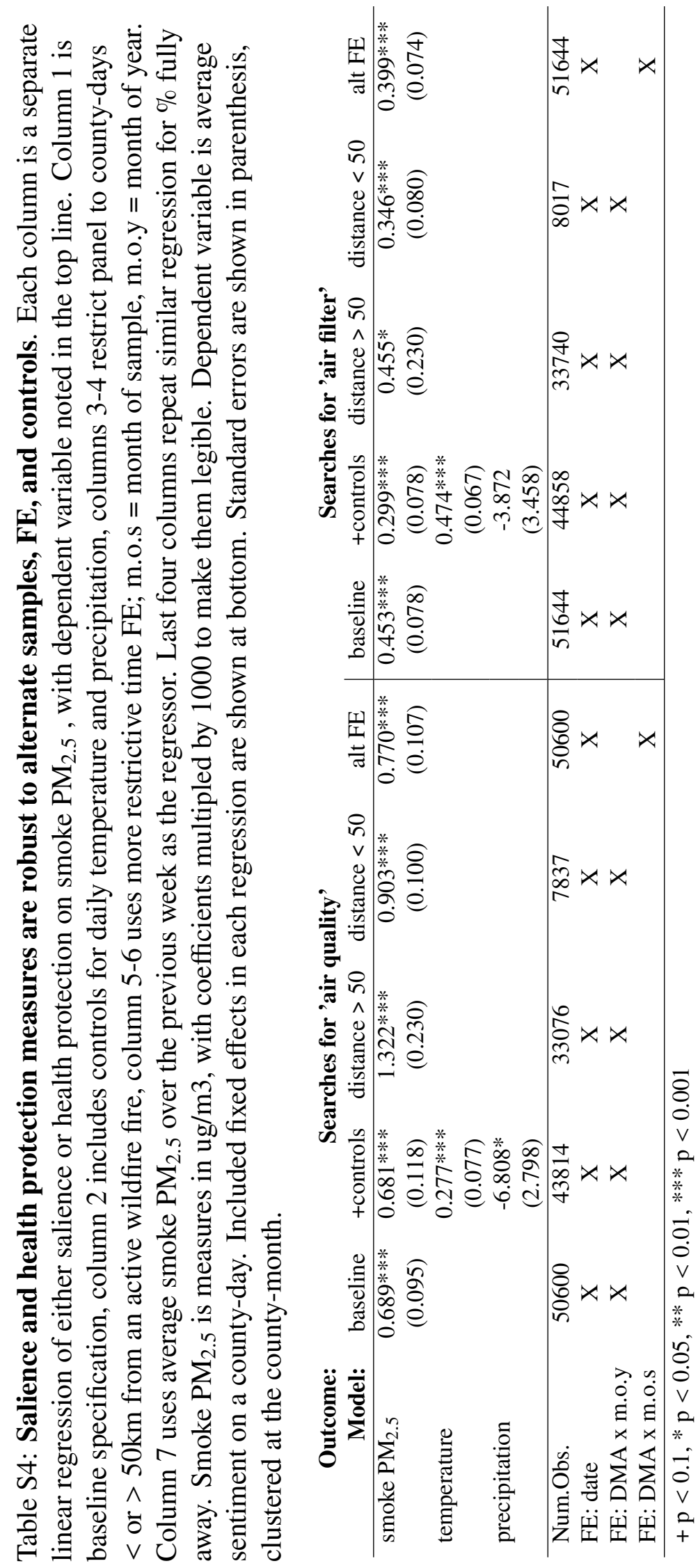


Table S5: Sentiment results are robust to alternate samples, FE, and controls. Each column is a separate linear regression of sentiment on smoke $\mathrm{PM}_{2.5}$. Column 1 is baseline specification, column 2 includes controls for daily temperature and precipitation, columns 3-4 restrict panel to county-days $<$ or $>50 \mathrm{~km}$ from an active wildfire fire, columns 5-6 uses more restrictive time FE; m.o.s $=$ month of sample, m.o.y $=$ month of year. Smoke $\mathrm{PM}_{2.5}$ is measures in ug/m3, with coefficients multipled by 1000 to make them legible. Dependent variable is average sentiment on a county-day. Included fixed effects in each regression are shown at bottom. Standard errors are shown in parenthesis, clustered at the county-month.

\begin{tabular}{|c|c|c|c|c|c|c|}
\hline Model: & baseline & + controls & distance $>50$ & distance $<50$ & alt FE & alt FE \\
\hline smoke $\mathrm{PM}_{2.5}$ & $\begin{array}{c}-0.087 * * * \\
(0.011)\end{array}$ & $\begin{array}{c}-0.088 * * * \\
(0.011)\end{array}$ & $\begin{array}{c}-0.085 * * * \\
(0.013)\end{array}$ & $\begin{array}{c}-0.081 * * * \\
(0.017)\end{array}$ & $\begin{array}{c}-0.075 * * * \\
(0.016)\end{array}$ & $\begin{array}{c}-0.082 * * * \\
(0.009)\end{array}$ \\
\hline temperature & & $\begin{array}{c}0.000 \\
(0.000)\end{array}$ & & & & \\
\hline precipitation & & $\begin{array}{c}-0.011 * * * \\
(0.000)\end{array}$ & & & & \\
\hline Num.Obs. & 1239533 & 1175623 & 1009505 & 211485 & 1239533 & 1239533 \\
\hline FE: date & $\mathrm{X}$ & $X$ & $\mathrm{X}$ & $X$ & & $X$ \\
\hline FE: date $x$ state & & & & & $\mathrm{X}$ & \\
\hline FE: county x m.o.y. & $\mathrm{X}$ & $\mathrm{X}$ & $\mathrm{X}$ & $X$ & $\mathrm{X}$ & \\
\hline FE: county x m.o.s. & & & & & & $\mathrm{X}$ \\
\hline
\end{tabular}


Table S6: Health protection results are largely robust to alternate search words in English and Spanish. Each column is a separate regression using the word in the column header as the search term. All models include the same fixed effects but different numbers of observations, as Google Trends does not return information for areas where search volume was low. Observations are marketing area by week. Standard errors are shown in parentheses, clustered at marketing area month.

\begin{tabular}{lcccccc}
\multicolumn{1}{c}{ Outcome: } & air filter & air purifier & purple air & smoke mask & purificador de aire & filtro de aire \\
\hline smokePM & $0.453^{* * *}$ & $0.540^{* * *}$ & $0.535^{* * *}$ & $0.350^{* * *}$ & $0.485^{* *}$ & 0.020 \\
& $(0.078)$ & $(0.069)$ & $(0.103)$ & $(0.089)$ & $(0.163)$ & $(0.102)$ \\
\hline Num.Obs. & 51644 & 50343 & 29493 & 8091 & 2088 & 4959 \\
FE: date & $\mathrm{X}$ & $\mathrm{X}$ & $\mathrm{X}$ & $\mathrm{X}$ & $\mathrm{X}$ & $\mathrm{X}$ \\
FE: DMA x month & $\mathrm{X}$ & $\mathrm{X}$ & $\mathrm{X}$ & $\mathrm{X}$ & $\mathrm{X}$ & $\mathrm{X}$ \\
\hline
\end{tabular}

$+\mathrm{p}<0.1, * \mathrm{p}<0.05, * * \mathrm{p}<0.01, * * * \mathrm{p}<0.001$ 


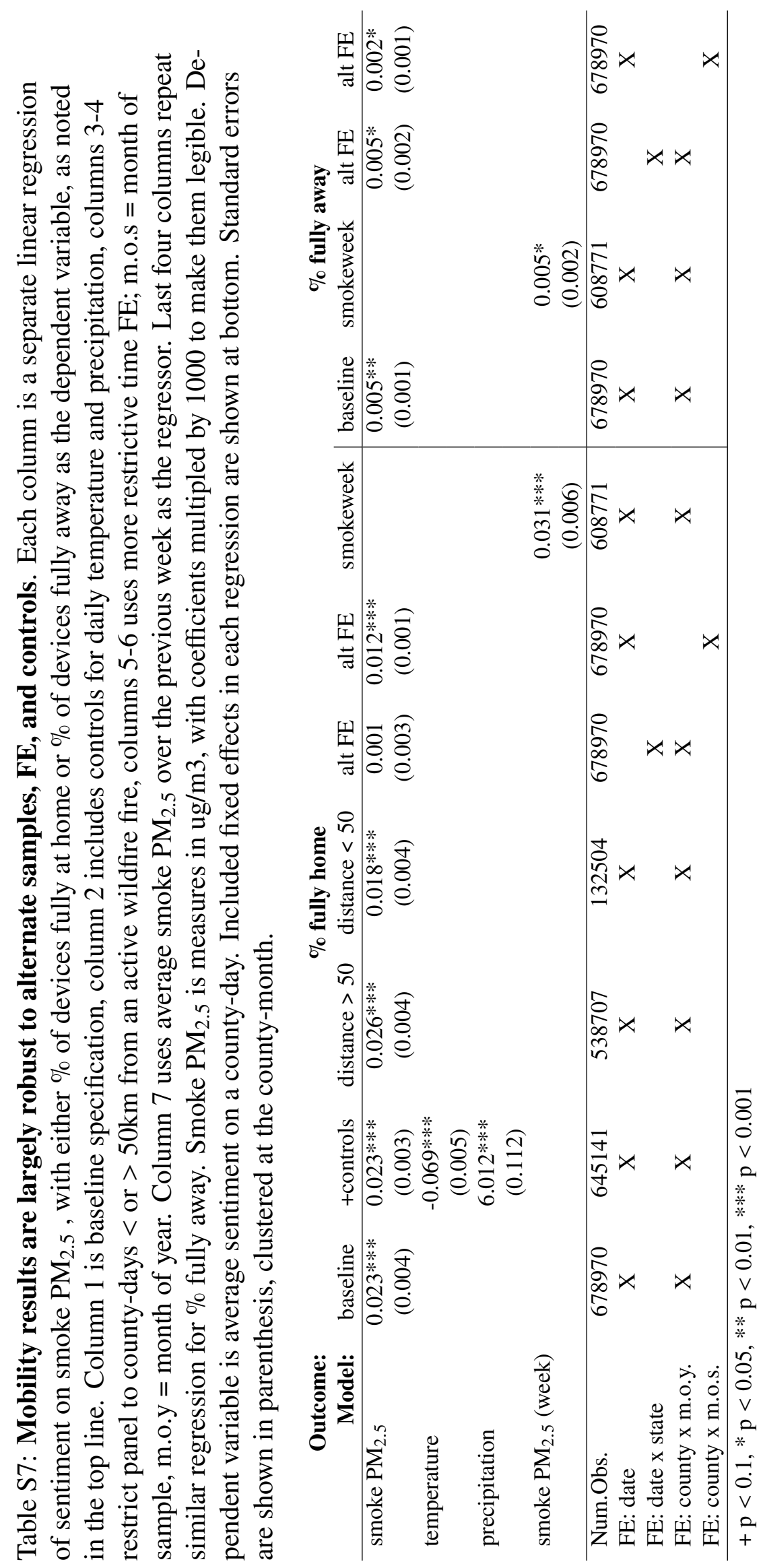




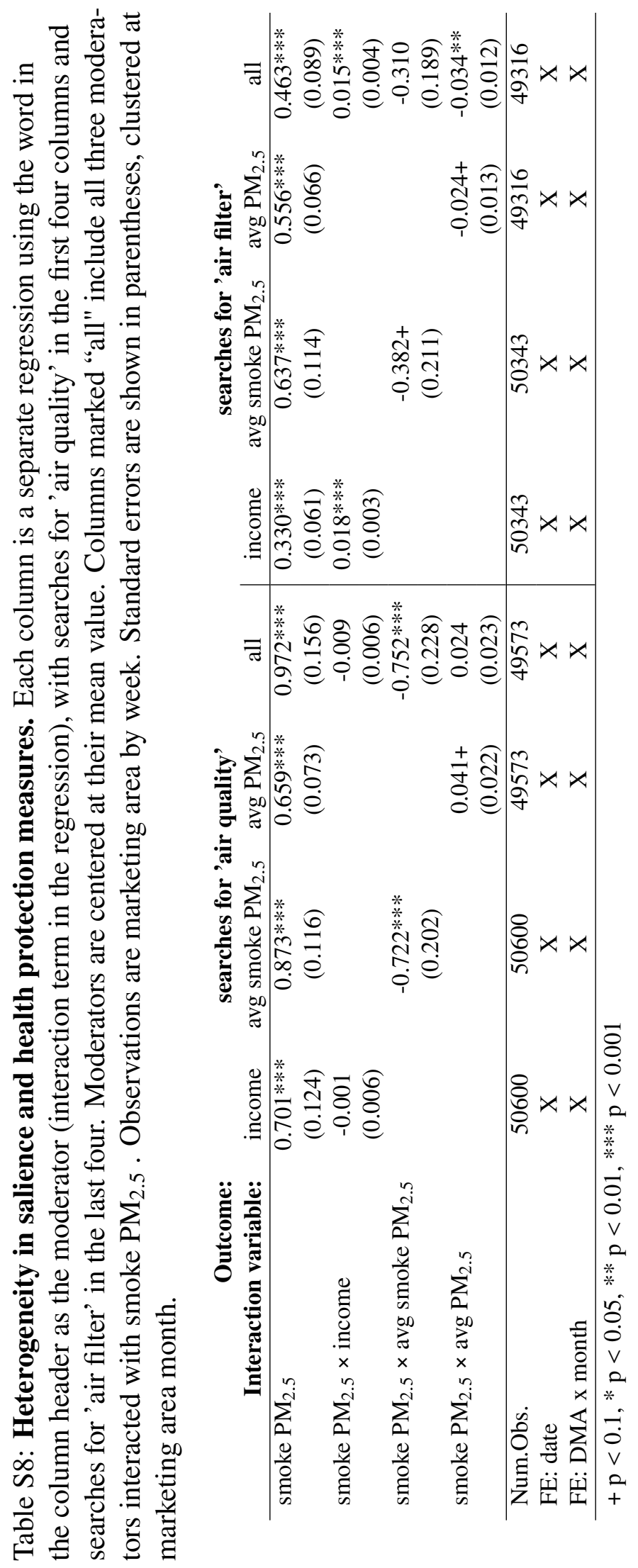




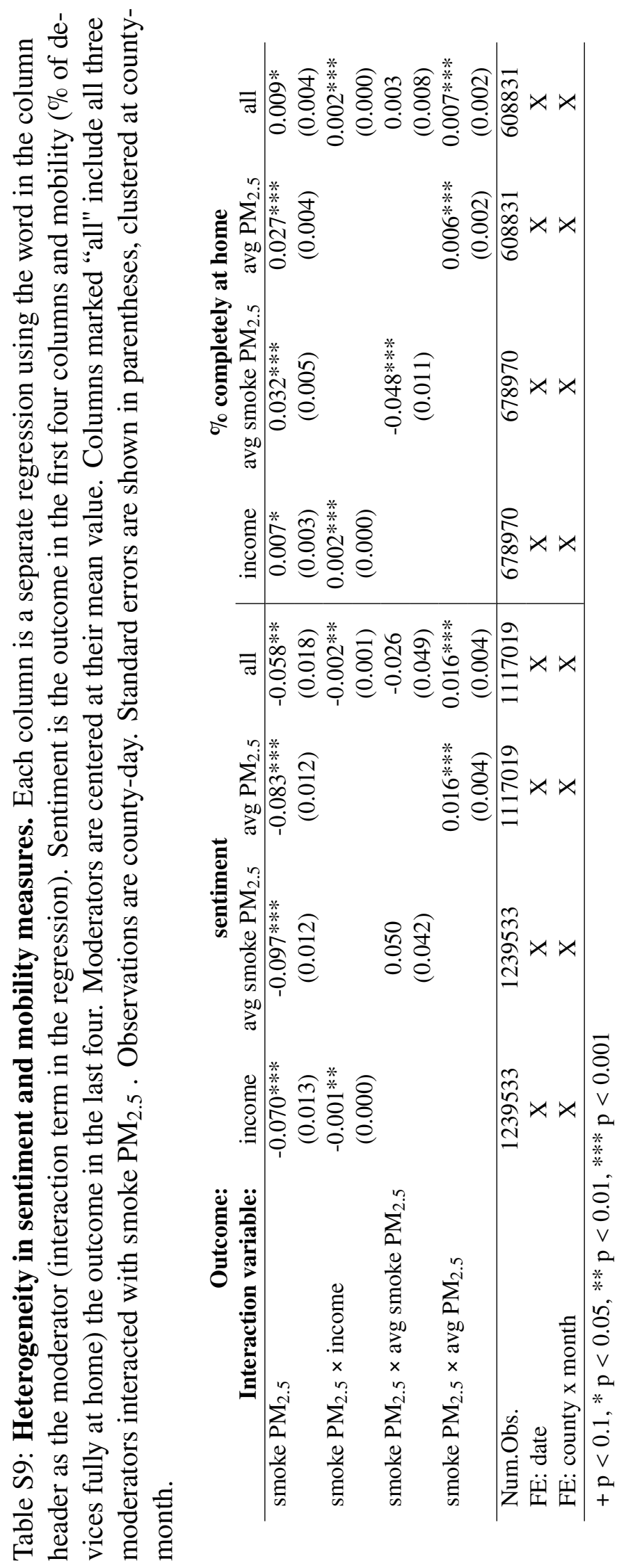

